\title{
Dual Action on a von Neumann Algebra and Takesaki's Duality for a Locally Compact Group
}

By

\author{
Yoshiomi NaKaGAMI*
}

\begin{abstract}
We define a dual action $\beta$ of a locally compact group $G$ on a von Neumann algebra $N$ and a crossed dual product $N \otimes_{\beta}^{d} G$. Then the Takesaki's duality is generalized in terms of these definitions as follows:

$$
\left(M \otimes_{\alpha} G\right) \otimes_{\hat{\alpha}}^{d} G \sim M \otimes B\left(L^{2}(G)\right),
$$
\end{abstract}

where $\hat{\alpha}$ is the dual action dual to a given action $\alpha$, and

$$
\left(N \otimes_{\beta}^{d} G\right) \otimes_{\hat{\beta}} G \sim N \otimes B\left(L^{2}(G)\right),
$$

where $\hat{\beta}$ is the action dual to a given dual action $\beta$. As an application

$$
M \otimes_{\alpha} G \sim M^{\alpha} \otimes B\left(L^{2}(G)\right)
$$

whenever $1 \otimes L^{\infty}(G) \subset M$ and $\alpha(1 \otimes f)=1 \otimes \varepsilon f$.

\section{Introduction}

The main purpose of this paper is to generalize the Takesaki's duality of crossed products for locally compact abelian groups to that for a non abelian one $[18,13]$.

To see the situation more precisely we shall prepare some results which are necessary for Takesaki's duality. We first notice that a necessary and sufficient condition for an isomorphism $\alpha$ of $M$ into $M \otimes$ $L^{\infty}(G)$ to be induced from an action is that $\alpha$ satisfies the commutative diagram:

Communicated by M. Sato, April 24, 1976.

* Department of Mathematics, Faculty of Science, Kyushu University, Fukuoka 812, Japan. 


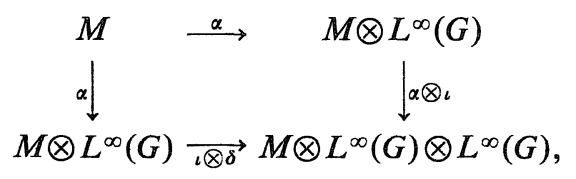

where $\iota$ is the identity automorphism and $\delta$ is given by (1.5), (Theorem 2.1). Let $R(G)$ be the von Neumann algebra generated by the regular representation of $G$ on $L^{2}(G)$. The crossed product $M \otimes_{\alpha} G$ is then defined as the von Neumann algebra generated by $\alpha(M)$ and $1 \otimes R(G)$. Here, we denote $M \otimes_{\alpha} G$ by $N$. Then the same diagram holds for the action $\hat{\alpha}$ dual to $\alpha$ of the dual group $\hat{G}$ on $N$ :

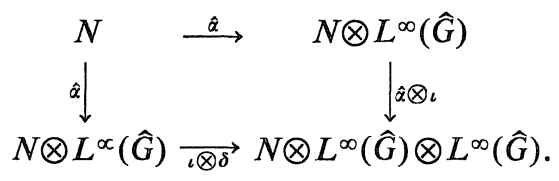

Making use of these $\alpha$ and $\hat{\alpha}$, we can state Takesaki's duality as follows:

$$
\left(M \otimes_{\alpha} G\right) \otimes_{\hat{\alpha}} \hat{G} \sim M \otimes B\left(L^{2}(G)\right),
$$

where $A \sim B$ means that $A$ is isomorphic to $B$. Let $F$ be the Fourier transformation of $L^{2}(\hat{G})$ onto $L^{2}(G)$ and $\beta$ a mapping of $N$ into $N$ $\otimes R(G)$ defined by $\beta \equiv(\operatorname{Ad} 1 \otimes F) \circ \hat{\alpha}$. Let $\lambda$ be the regular representation of $G$ on $L^{2}(G)$ and $\gamma$ a mapping given by (1.5). Since

$$
(\operatorname{Ad} F \otimes F) \circ \delta=\gamma \circ \operatorname{Ad} F
$$

by (1.8), $\beta$ satisfies

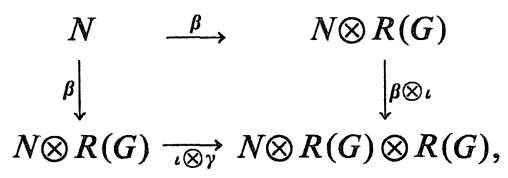

and $\hat{\alpha}$ coincides with $\beta$ up to the spatial isomorphism $\operatorname{Ad} 1 \otimes F$. We shall call an isomorphism satisfying the commutative diagram (*) a dual action. $\beta$ is then a dual action which is dual to $\alpha_{0}$ By using a dual action we shall define a crossed dual product $N \otimes_{\beta}^{d} G$ of $N$ by $G$ as the von Neumann algebra

$$
\{\beta(N), 1 \otimes R(G)\}^{\prime \prime} \quad\left(=\operatorname{Ad} 1 \otimes F\left(N \otimes_{\hat{\alpha}} \hat{G}\right)\right),
$$


(Definition 2.2). Then the Takesaki's duality is restated as follows:

$$
\left(M \otimes_{\alpha} G\right) \otimes_{\beta}^{d} G \sim M \otimes B\left(L^{2}(G)\right) .
$$

Changing the roles of $\{\alpha, G\}$ and $\{\hat{\alpha}, \hat{G}\}$ and applying the Fourier transformation, we have

$$
\left(N \otimes{ }_{\beta}^{d} G\right) \otimes_{\hat{\beta}} G \sim N \otimes B\left(L^{2}(G)\right),
$$

where $\hat{\beta}$ is the action dual to $\beta$, (Theorem 2.3).

Since Theorem 2.1 holds for a general locally compact group as well, the diagram $(*)$ and the crossed dual product $(* *)$ have their meanings even when $G$ is not necessarily abelian. Therefore our generalizations are obtained in the same forms as above in Theorems 3.1 and 7.1. The contents of this paper is the following:

0 . Introduction

1. Preliminary

2. Dual action $\beta$ and crossed dual product

3. Duality for crossed product by $\alpha$.

4. Some technical lemmas for $\beta$

5. Spectrum of $\beta$

6. Fixed points of $\alpha$ and $\beta$

7. Duality for crossed dual product by $\beta$

8. Haga's factorization of crossed product

9. Appendix.

Here, the reader who wants to know directly the Takesaki's duality of the second type, can skip Sections 5 and 6, which are prepared only for Corollaries 7.4, 7.5 and Section 8. In Section 8 we shall give a sufficient condition for a crossed product $M \otimes_{\alpha} G$ and a crossed dual product $N \otimes{ }_{\beta}^{d} G$ to be factorized into $M^{\alpha} \otimes B\left(L^{2}(G)\right)$ and $N^{\beta} \otimes B\left(L^{2}(G)\right)$, respectively, by using the idea of Landstad, [9], (Theorems 8.4 and 8.2).

Recently, Roberts [14] has obtained interesting results which have close connection with ours.

The author wants to express his deep gratitude to Professor M. Takesaki for his valuable discussion and Professor M. Tomita for his encouragement. 


\section{Preliminary}

Let $G$ be a locally compact group, $d t$ the right invariant Haar measure and $I(f)$ the integral

$$
\int f(t) d t
$$

The modular function $\Delta$ satisfies $\Delta(t) I(f)=I\left(f_{t}\right)$ for $f \in L^{1}(G)$, where $f_{t}(s) \equiv f(t s)$. Let $\lambda$ be the right regular representation of $G$ on $L^{2}(G)$, $\lambda(f)$ for $f$ in $L^{1}(G)$ the integral

$$
\int f(t) \lambda(t) d t
$$

and $R(G)$ the von Neumann algebra generated by $\lambda(G)$ or $\lambda\left(L^{1}(G)\right)$.

When $G$ is abelian, the spectrum $\hat{G}$ of $L^{1}(G)$ becomes a locally compact abelian group and the spectrum of $L^{1}(\hat{G})$ is isomorphic to $G$. For $\xi, \eta$ in $L^{2}(G)$ and $f$ in $L^{1}(G)$, using Plancherel theorem, we have

$$
\begin{aligned}
\omega_{\xi, \eta}(\lambda(f)) & =\int_{G} f(t) \omega_{\xi, \eta}(\lambda(t)) d t \\
& =\int_{G} f(t)(\tilde{\eta} * \xi)(t) d t \\
& =\int_{\hat{G}} \hat{f}(\zeta) \hat{\xi}(\zeta) \hat{\tilde{\eta}}(\zeta) d \zeta,
\end{aligned}
$$

where $*$ denotes the convolution, $\tilde{\eta}(t) \equiv \overline{\eta\left(t^{-1}\right)}$ and $d \zeta$ denotes the Haar measure on $\hat{G}$ associated with $d t$. We identify $R(G)$ and $R(G)_{*}$ with $L^{\infty}(\hat{G})$ and $L^{1}(\hat{G})$, respectively, through the correspondence in (1.1):

$$
\begin{aligned}
& \lambda(f) \in R(G) \longleftrightarrow \hat{f} \in L^{\infty}(\hat{G}) \\
& \omega_{\xi, \eta} \in R(G)_{*} \longleftrightarrow \hat{\xi} \hat{\tilde{\eta}} \in L^{1}(\hat{G}) .
\end{aligned}
$$

Then the duality between $G$ and $\hat{G}$ is expressed by $\lambda$ as the following diagram:

$$
\begin{aligned}
& L^{1}(G) \stackrel{\lambda}{\longrightarrow} R(G) \\
& L^{\infty}(G) \stackrel{\leftarrow}{\lambda_{*}} R(G)_{*}
\end{aligned}
$$


where $\lambda_{*}$ denotes the dual of $\lambda$ to $R(G)_{*} . \quad \lambda$ is therefore considered as the Fourier transformation through the above identification (1.2).

For a non abelian $G$, the duality theorem for $G$ by Eymard, Takesaki and Saito $[5,17,15]$ has the same diagram as (1.3). Let $K(G)$ denote the set of all continuous functions with compact carrier on $G$ and $B(G)$ the set of all limits relative to compact convergence of finite linear combinations of functions of positive type on $G$ with respect to $\lambda$. Define a norm of $g$ in $B(G)$ by

$$
\sup \left\{|(f \mid g)|: f \in L^{1}(G),\|\lambda(f)\| \leq 1\right\}<\infty .
$$

$B(G)$ is a commutative Banach algebra. The mapping $f \mapsto \tilde{f}$ gives an involution in $B(G)$. Denote the closed lincar span of

$$
\{\tilde{g} * f: f, g \in K(G)\} \quad\left((\tilde{g} * f)(t) \equiv \int \tilde{g}\left(t s^{-1}\right) f(s) d s\right)
$$

in $B(G)$ by $A(G)$, which is called the Fourier algebra of $G$. It is known that $A(G)$ is a regular, semi-simple, abelian and involutive Banach algebra and coincides with the set of all $\tilde{\eta} * \xi$ with $\xi, \eta$ in $L^{2}(G)$. Since for $\xi$ and $\eta$ in $L^{2}(G)$

$$
(\lambda(s) \xi \mid \eta)=(\tilde{\eta} * \xi)(s)
$$

as in (1.1), $\lambda_{*}\left(\omega_{\xi, \eta}=\tilde{\eta} * \xi\right.$ and $A(G)=\lambda_{*} R(G)_{*}$. Therefore $R(G)_{*}$ has the same algebraic structure as $A(G)$. Denote the product of $\phi$ and $\psi$ in $R(G)_{*}$ by $\phi \psi$, that is, $\lambda_{*}(\phi \psi)=\left(\lambda_{*} \phi\right)\left(\lambda_{*} \psi\right)$. For a non zero $y$ in $R(G)$ the following two conditions are equivalent:

(i) $y=\lambda(t)$ for some $t \in G$; and

(ii) $\langle y, \phi \psi\rangle=\left\langle y, \phi>\langle y, \psi\rangle\right.$ for all $\phi, \psi \in R(G)_{*}$.

In what follows we shall identify the spectrum (the set of characters) of $A(G)$ with the original $G$ through $\lambda$.

Now we define two mappings

$$
\begin{aligned}
& \delta: L^{\infty}(G) \longrightarrow L^{\infty}(G) \otimes L^{\infty}(G) \\
& \gamma: R(G) \longrightarrow R(G) \otimes R(G)
\end{aligned}
$$

by

$$
(\delta f)(s, t) \equiv f(s t), \quad \gamma \lambda(s) \equiv \lambda(s) \otimes \lambda(s) .
$$


Then by (ii) we have

$$
<\gamma \lambda(t), \phi \otimes \psi>=<\lambda(t), \phi \psi>
$$

In case of an abelian $G$, if $f$ is an element of $L^{1}(G)$,

$$
\begin{aligned}
<\gamma \lambda(f), \phi \otimes \psi> & =\int_{G} f(t)\langle\gamma \lambda(t), \phi \otimes \psi\rangle d t \\
& =\int_{G} f(t)\left(\lambda_{*} \phi\right)(t)\left(\lambda_{*} \psi\right)(t) d t \\
& =\int_{\hat{G}} \hat{f}(\zeta)\left(\lambda_{*} \phi\right)^{\wedge} *\left(\lambda_{*} \psi\right)^{\wedge}(\zeta) d \zeta \\
& =\int_{\hat{G}} \int_{\hat{G}} \hat{f}\left(\zeta \zeta^{\prime}\right)\left(\lambda_{*} \phi\right)^{\wedge}(\zeta)\left(\lambda_{*} \psi\right)^{\wedge}\left(\zeta^{\prime}\right) d \zeta d \zeta^{\prime} \\
& =<\delta \hat{f},\left(\lambda_{*} \phi\right)^{\wedge} \otimes\left(\lambda_{*} \psi\right)^{\wedge}>,
\end{aligned}
$$

where $d \zeta$ and $d \zeta^{\prime}$ are the Haar measure on $G$ associated with $d t$. Therefore, under the identification of $R(G)$ with $L^{\infty}(\hat{G})$ as in (1.2), we have

$$
\gamma \lambda(f)=\delta \hat{f}
$$

with which we combine the argument in Introduction, we shall define a crossed dual product in Section 2.

The following four unitary operators on $L^{2}(G) \otimes L^{2}(G)$ play important roles in our paper:

$$
\begin{aligned}
& (W \xi)(s, t) \equiv \xi(s, t s), \quad\left(W^{\prime} \xi\right)(s, t) \equiv \Delta(s)^{1 / 2} \xi\left(s, s^{-1} t\right) \\
& (V \xi)(s, t) \equiv \xi(s t, t), \quad\left(V^{\prime} \xi\right)(s, t) \equiv \Delta(t)^{1 / 2} \xi\left(t^{-1} s, t\right) .
\end{aligned}
$$

Let 1 (resp. $1_{G}$ ) be the identity operator on a Hilbert space $\mathscr{H}$ or $\mathscr{K}$ (resp. $L^{2}(G)$ ). Let $\lambda^{\prime}$ denote the left regular representation of $G$ on $L^{2}(G)$ :

$$
\left(\lambda^{\prime}(s) \xi\right)(t) \equiv \Delta(s)^{1 / 2} \xi\left(s^{-1} t\right), \quad \xi \in L^{2}(G)
$$

and

$$
\begin{aligned}
& \lambda_{1}(t) \equiv 1 \otimes \lambda(t), \quad \lambda_{2}(t) \equiv 1 \otimes 1_{G} \otimes \lambda(t) \\
& \lambda_{1}^{\prime}(t) \equiv 1 \otimes \lambda^{\prime}(t), \quad \lambda_{2}^{\prime}(t) \equiv 1 \otimes 1_{G} \otimes \lambda^{\prime}(t) .
\end{aligned}
$$


When a measure $\mu$ converges to a Dirac measure $\varepsilon_{e}$ at the unit $e$ of $G$ in the dual space of $C(G)$ with the compact convergence topology, we say simply that $\mu$ converges to $\varepsilon_{e}$ in this paper. For example, let $\mathscr{U}$ be a compact symmetric neighbourhood of $e,\{\mathscr{V}\}$ a fundamental system of compact symmeteric neighbourhoods of $e$ satisfying $\mathscr{V}^{2} \subset \mathscr{U}$ and $g_{\mathscr{V}} \equiv \tilde{\chi}_{\mathscr{V}} * \chi_{\mathscr{V}} /\left\|\tilde{\chi}_{\mathscr{V}} * \chi_{\mathscr{V}}\right\|_{1}$, where $\chi_{\mathscr{V}}$ denotes the indicator function of $\mathscr{V}$. Then $g_{\mathscr{V}} \in P(G) \cap K(G)_{+}$and a measure $g_{\mathscr{V}}(t) d t$ converges to $\varepsilon_{\boldsymbol{e}}$, where $P(G) \equiv \lambda_{*} R(G)_{*}^{+}$.

\section{Dual Action $\beta$ and Crossed Dual Product}

In this section we shall define a dual action and a crossed dual product for our later Sections.

Let $M$ be a von Neumann algebra on a Hilbert space $\mathscr{H}$ and Aut $M$ the automorphism group of $M$. By an action of $G$ on $M$ we mean a homomorphism $\sigma: t \in G \mapsto \sigma_{t} \in$ Aut $M$ such that for each $x$ in $M$ the mapping $t \in G \mapsto \sigma_{t}(x) \in M$ is $\sigma$-strongly* continuous. Let $\left\{\pi_{\sigma}, \lambda_{1}\right\}$ be a covariant representation of $\{M, \sigma\}$ on $\mathscr{H} \otimes L^{2}(G)$ defined by

$$
\begin{aligned}
& \left(\pi_{\sigma}(x) \xi\right)(s) \equiv \sigma_{s}(x) \xi(s) \\
& \left(\lambda_{1}(r) \check{\zeta}\right)(s) \equiv \xi(s r)
\end{aligned}
$$

for $\xi \in \mathscr{H} \otimes L^{2}(G)$. The crossed product $M \otimes_{\sigma} G$ of $M$ by $G$ is the von Neumann algebra gencrated by $\pi_{\sigma}(M)$ and $\lambda_{1}(G)$.

Since $M \otimes L^{\infty}(G)$ is isomorphic to the sct $L^{\infty}(G, M)$ of all essentially bounded $M$-valued $\sigma$-weakly measurable functions on $G$ by $[12,16]$, $\pi_{\sigma}(x)$ is identified with a function $s \mapsto \sigma_{s}(x)$ in $L^{\infty}(G, M)$.

Theorem 2.1. A necessary and sufficient condition that a mapping $\alpha$ of $M$ into $M \otimes L^{\infty}(G)$ be induced by an action $\sigma$ with

$$
(\alpha(x) \xi)(s)=\sigma_{s}(x) \xi(s)
$$

is that $\alpha$ be an isomorphism which satisfies

$$
(\alpha \otimes \iota) \circ \alpha=(\iota \otimes \delta) \circ \alpha .
$$

Proof. Necessity. Sincc $\alpha$ is known to be an isomorphism, we 
have only to show (2.2). Since $\alpha(x)$ is an essentially bounded $\sigma$-weakly measurable function

$$
s \in G \longmapsto \sigma_{s}(x) \in M,
$$

$(\alpha \otimes \iota) \alpha(x)$ and $(\iota \otimes \delta) \alpha(x)$ correspond respectively essentially bounded $\sigma$-weakly measurable functions

$$
(s, t) \in G \times G \longmapsto \sigma_{s}\left(\sigma_{t}(x)\right) \in M
$$

and

$$
(s, t) \in G \times G \longmapsto \sigma_{s t}(x) \in M
$$

Since $\sigma$ is an action by hypothesis, these two functions coincide and hence (2.2) follows.

Sufficiency. We shall begin by showing that $\tau_{r} \equiv \operatorname{Ad} \lambda_{1}(r) \uparrow \alpha(M)$, $r \in G$ is an action on $\alpha(M)$. Put $L \equiv \alpha(M)$ and $\bar{\delta} \equiv \iota \otimes \delta$. Since

$$
\begin{aligned}
\bar{\delta}(L) & =(\alpha \otimes \iota) \alpha(M) \\
& \subset\left(\alpha \otimes_{\ell}\right)\left(M \otimes L^{\infty}(G)\right)=L \otimes L^{\infty}(G)
\end{aligned}
$$

and since

$$
\left|<\bar{\delta}(z), \omega^{\prime} \otimes g>\right| \leq\|z\|\left\|\omega^{\prime}\right\|\|g\|_{1}
$$

for each $z \in L, \omega^{\prime} \in L_{*}$ and $g \in L^{1}(G)$, we can define a bounded linear operator $\bar{\delta}_{g}$ on $L$ by

$$
<\bar{\delta}_{g}(z), \omega^{\prime}>=<\delta(z), \omega^{\prime} \otimes g>.
$$

If $y \in L$, then for any $\omega$ defined by vectors in $\mathscr{H}$ and $f, g \in K(G)$ we have

$$
\begin{aligned}
<\bar{\delta}_{g}(y), \omega \otimes f> & =\iint \omega(y(s t)) f(s) g(t) d s d t \\
& =\int \omega(y(s)) f * g(s) d s
\end{aligned}
$$

Making the measure $g(t) d t$ converge to the Dirac measure $\varepsilon_{r}$ at $r \in G$, we know that the right hand side converges to 


$$
\begin{aligned}
\int \omega(y(s)) f\left(s r^{-1}\right) d s & =\int \omega(y(s r)) f(s) d s \\
& =<\tau_{r}(y), \omega \otimes f>
\end{aligned}
$$

In the above convergence we may assume that $\|g\|_{1} \leq 1$ and hence $\left\|\bar{\delta}_{g}(y)\right\| \leq\|y\|$. Further, $\omega \otimes f$ are total in the predual of $L$. Indeed, since the convex hull of all $\omega \otimes f$ is weakly dense in $L_{*}$, it is also norm dense by the Hahn-Banach's separation theorem. Therefore, $\bar{\delta}_{g}(y)$ converges $\sigma$-weakly to $\tau_{r}(y)$. Since $\bar{\delta}_{g}(y) \in L$ from the above, $\tau_{r}(y) \in L$. Since $\tau_{r}^{-1}=\tau_{r^{-1}}, \tau_{r}(L)=L$. Since $\operatorname{Ad} \lambda_{1}(r)$ is an isomorphism and $r \mapsto$ $\operatorname{Ad} \lambda_{1}(r)(z)$ is $\sigma$-strongly* continuous for cach $z \in M \otimes L^{\infty}(G)$, its restriction $\tau_{r}$ to $L$ is an action of $G$ on $L$.

Now we define an action $\sigma$ of $G$ on $M$ by

$$
\sigma_{s} \equiv \alpha^{-1} \circ \tau_{s} \circ \alpha
$$

We shall show $\alpha=\pi_{\sigma}$. For this we define two bounded linear operators $\alpha_{g}$ and $\sigma(g)$ on $M$ for $g \in L^{1}(G)$ by

$$
<\alpha_{g}(x), \omega>=<\alpha(x), \omega \otimes g>
$$

for $x \in M$ and $\omega \in M_{*}$, and

$$
\sigma(g) \equiv \int g(s) \sigma_{s} d s
$$

If $\omega \in M_{*}$ and $f \in L^{1}(G)$, then

$$
\begin{aligned}
& <\alpha(\sigma(g) x), \omega \otimes f> \\
& =\int g(t)<\alpha \circ \sigma_{t}(x), \omega \otimes f>d t \\
& =\int g(t)<\tau_{t} \circ \alpha(x), \omega \otimes f>d t \\
& =\int g(t)<\alpha(x), \omega \otimes_{t^{-1}} f>d t \\
& =<\alpha(x), \omega \otimes(f * g)> \\
& =<\left({ }_{\iota} \otimes \delta\right) \alpha(x), \omega \otimes f \otimes g>
\end{aligned}
$$




$$
\begin{aligned}
& =<(\alpha \otimes \imath) \alpha(x), \omega \otimes f \otimes g> \\
& =<\alpha(x), \alpha_{*}(\omega \otimes f) \otimes g> \\
& =<\alpha\left(\alpha_{g}(x)\right), \omega \otimes f>
\end{aligned}
$$

where ${ }_{r} f(s) \equiv f(s r)$ and $\alpha_{*}$ is the dual mapping of $\alpha$. Since $\omega$ and $f$ are arbitrary and $\alpha$ is an isomorphism, we have $\sigma(g) x=\alpha_{g}(x)$. Therefore

$$
\begin{aligned}
<\pi_{\sigma}(x), \omega \otimes g>= & =\sigma(g) x, \omega> \\
= & <\alpha_{g}(x), \omega>=\langle\alpha(x), \omega \otimes g>.
\end{aligned}
$$

Since $\omega$ and $g$ are arbitrary, $\alpha(x)=\pi_{\sigma}(x)$.

Q.E.D.

From this theorem we can identify an isomorphism of $M$ into $M \otimes L^{\infty}(G)$ satisfying (2.2) with an action of $G$ on $M$. Therefore we shall use the same letter for them.

Definition 2.2. A dual action $\beta$ of $G$ on $N$ is an isomorphism of a von Neumann algebra $N$ into $N \otimes R(G)$ satisfying

$$
(\beta \otimes \iota) \circ \beta=(\iota \otimes \gamma) \circ \beta
$$

A crossed dual product of $N$ by $G$ with respect to $\beta$ is the von Neumann algebra generated by $\beta(N)$ and $1 \otimes L^{\infty}(G)$, which is denoted by $N \otimes \otimes_{\beta}^{d} G$.

Theorem 2.3. (i) Let $\sigma^{W}$ be an isomorphism of $B\left(\mathscr{H} \otimes L^{2}(G)\right)$ into $B\left(\mathscr{H} \otimes L^{2}(G) \otimes L^{2}(G)\right)$ defined by

$$
\sigma^{W}(y) \equiv \operatorname{Ad} 1 \otimes W^{*}\left(y \otimes 1_{G}\right) .
$$

If $\alpha$ is an action of $G$ on $M$, then $\hat{\alpha} \equiv \sigma^{W} \uparrow M \otimes_{\alpha} G$ is a dual action of $G$ on $M \otimes_{\alpha} G$.

(ii) Let $N$ be a von Neumann algebra on a Hilbert space $\mathscr{K}$ and $\sigma^{V^{\prime}}$ an isomorphism of $B\left(\mathscr{K} \otimes L^{2}(G)\right)$ into $B\left(\mathscr{K} \otimes L^{2}(G) \otimes L^{2}(G)\right)$ defined by

$$
\sigma^{V^{\prime}}(z) \equiv \operatorname{Ad} 1 \otimes V^{\prime}\left(z \otimes 1_{G}\right)
$$


If $\beta$ is a dual action of $G$ on $N$, then $\hat{\beta} \equiv \sigma^{V^{\prime}} \uparrow N \otimes_{\beta}^{d} G$ is an action of $G$ on $N \otimes_{\beta}^{d} G$.

Proof. (i) If $x \in M$ and $\xi \in \mathscr{H} \otimes L^{2}(G) \otimes L^{2}(G)$, then

$$
\begin{aligned}
\left((1 \otimes W)^{*}\left(\alpha(x) \otimes 1_{G}\right)(1 \otimes W) \xi\right)(s, t) \\
\quad=\left(\left(\alpha(x) \otimes 1_{G}\right)(1 \otimes W) \xi\right)\left(s, t s^{-1}\right) \\
\quad=\alpha_{s}(x)((1 \otimes W) \xi)\left(s, t s^{-1}\right) \\
\quad=\alpha_{s}(x) \xi(s, t)=\left(\left(\alpha(x) \otimes 1_{G}\right) \xi\right)(s, t)
\end{aligned}
$$

and

$$
\begin{aligned}
\left((1 \otimes W)^{*}\left(\lambda_{1}(r) \otimes 1_{G}\right)(1 \otimes W) \xi\right)(s, t) \\
\quad=\left(\left(\lambda_{1}(r) \otimes 1_{G}\right)(1 \otimes W) \xi\right)\left(s, t s^{-1}\right) \\
\quad=((1 \otimes W) \xi)\left(s r, t s^{-1}\right)=\xi(s r, t r) \\
\quad=\left(\left(\lambda_{1}(r) \otimes \lambda(r)\right) \xi\right)(s, t) .
\end{aligned}
$$

Therefore

$$
\hat{\alpha}(\alpha(x))=\alpha(x) \otimes 1_{G} \quad \text { and } \quad \hat{\alpha}\left(\lambda_{1}(r)\right)=\lambda_{1}(r) \otimes \lambda(r) .
$$

Since $M \otimes_{\alpha} G$ is generated by $\alpha(M)$ and $\lambda_{1}(G), \hat{\alpha}$ is a mapping of $M \otimes_{\alpha} G$ into $\left(M \otimes_{\alpha} G\right) \otimes R(G)$. It is clear that $\hat{\alpha}$ is an isomorphism. Since

$$
\begin{aligned}
& ((\hat{\alpha} \otimes \iota) \circ \hat{\alpha}) \alpha(x)=(\hat{\alpha} \otimes \iota)\left(\alpha(x) \otimes 1_{G}\right) \\
& \quad=\alpha(x) \otimes 1_{G} \otimes 1_{G}=(\iota \otimes \gamma)\left(\alpha(x) \otimes 1_{G}\right) \\
& \quad=((\iota \otimes \gamma) \circ \hat{\alpha}) \alpha(x)
\end{aligned}
$$

and

$$
\begin{aligned}
& ((\hat{\alpha} \otimes \iota) \circ \hat{\alpha}) \lambda_{1}(r)=(\hat{\alpha} \otimes \iota)\left(\lambda_{1}(r) \otimes \lambda(r)\right) \\
& \quad=\lambda_{1}(r) \otimes \lambda(r) \otimes \lambda(r)=(\iota \otimes \gamma)\left(\lambda_{1}(r) \otimes \lambda(r)\right) \\
& \quad=((\iota \otimes \gamma) \circ \hat{\alpha}) \lambda_{1}(r),
\end{aligned}
$$

(2.6) holds for $M \otimes_{\alpha} G$ and $\hat{\alpha}$. 
(ii) The argument will proceed similarly as (i). For each $f$ in $L^{\infty}(G)$ we define $T_{1}(f)$ and $\varepsilon f$ by

$$
T_{1}(f) \equiv 1 \otimes f
$$

on $\mathscr{K} \otimes L^{2}(G)$ and

$$
(\varepsilon f)(s, t) \equiv f\left(t^{-1} s\right)
$$

Since $\beta(N) \subset N \otimes R(G)$, it follows from (1.9) that $\left[\beta(y) \otimes 1_{G}, 1 \otimes V^{\prime}\right]=0$ for all $y$ in $N$. Since

$$
\begin{aligned}
& \left(\left(1 \otimes V^{\prime}\right)\left(T_{1}(f) \otimes 1_{G}\right)\left(1 \otimes V^{\prime}\right)^{*} \xi\right)(s, t) \\
& \quad=\Delta(t)^{1 / 2}\left(\left(T_{1}(f) \otimes 1_{G}\right)\left(1 \otimes V^{\prime}\right)^{*} \xi\right)\left(t^{-1} s, t\right) \\
& \quad=\Delta(t)^{1 / 2} f\left(t^{-1} s\right)\left(\left(1 \otimes V^{\prime}\right)^{*} \xi\right)\left(t^{-1} s, t\right) \\
& \quad=f\left(t^{-1} s\right) \xi(s, t)=((\varepsilon f) \xi)(s, t)
\end{aligned}
$$

for $\xi \in \mathscr{K} \otimes L^{2}(G) \otimes L^{2}(G)$, we have

$$
\hat{\beta}(\beta(y))=\beta(y) \otimes 1_{G} \quad \text { and } \quad \hat{\beta}\left(T_{1}(f)\right)=1 \otimes \varepsilon f
$$

for all $y \in N$ and $f \in L^{\infty}(G)$. Since

$$
\begin{aligned}
& ((\hat{\beta} \otimes \iota)(1 \otimes \varepsilon f))(s, t, r)=(1 \otimes \varepsilon f)\left(t^{-1} s, r\right) \\
& \quad=(1 \otimes f)\left(r^{-1} t^{-1} s\right)=(1 \otimes \varepsilon f)(s, t r) \\
& \quad=((\iota \otimes \delta)(1 \otimes \varepsilon f))(s, t, r),
\end{aligned}
$$

we have

$$
\begin{aligned}
& ((\hat{\beta} \otimes \iota) \circ \hat{\beta})\left(T_{1}(f)\right)=(\hat{\beta} \otimes \iota)(1 \otimes \varepsilon f) \\
& =(\iota \otimes \delta)(1 \otimes \varepsilon f)=((\iota \otimes \delta) \circ \hat{\beta})\left(T_{1}(f)\right) .
\end{aligned}
$$

Moreover, since

$$
\begin{aligned}
& ((\hat{\beta} \otimes \iota) \circ \hat{\beta})(\beta(y))=(\hat{\beta} \otimes \iota)\left(\beta(y) \otimes 1_{G}\right) \\
& \quad=\beta(y) \otimes 1_{G} \otimes 1_{G}=(\iota \otimes \delta)\left(\beta(y) \otimes 1_{G}\right) \\
& \quad=((\iota \otimes \delta) \circ \hat{\beta})(\beta(y))
\end{aligned}
$$


for all $y \in N,(2.2)$ holds for $N \otimes_{\beta}^{d} G$ and $\hat{\beta}$.

Q.E.D.

Definition 2.4. A dual action $\hat{\alpha}$ (resp. an action $\hat{\beta}$ ) in Theorem 2.3 is said to be dual to $\alpha$ (resp. $\beta$ ).

Let $\alpha^{j}$ be an action of $G$ on $M_{j}(j=1,2)$. When an isomorphism $\rho$ of $M_{1}$ onto $M_{2}$ satisfies

$$
(\rho \otimes \iota) \circ \alpha^{1}=\alpha^{2} \circ \rho \quad\left(\text { or } \rho \circ \alpha_{t}^{1}=\alpha_{t}^{2} \circ \rho\right)
$$

$\left\{M_{1}, \alpha^{1}\right\}$ and $\left\{M_{2}, \alpha^{2}\right\}$ are said to be equivalent. In this case, $M_{1} \otimes_{\alpha^{1}} G$ is isomorphic to $M_{2} \otimes_{\alpha^{2}} G$.

Definition 2.5. Let $\beta_{j}$ be a dual action of $G$ on $N_{j} \quad(j=1,2)$. $\left\{N_{1}, \beta_{1}\right\}$ and $\left\{N_{2}, \beta_{2}\right\}$ are said to be equivalent if there is an isomorphism $\rho$ of $N_{1}$ onto $N_{2}$ satisfying

$$
(\rho \otimes \iota) \circ \beta_{1}=\beta_{2} \circ \rho
$$

Of course, $N_{1} \otimes_{\beta_{1}}^{d} G$ is isomorphic to $N_{2} \otimes_{\beta_{2}}^{d} G$.

\section{Duality for Crossed Product by $a$}

We are now ready to show the following duality theorem for crossed products of von Neumann algebras by a locally compact group.

Theorem 3.1. Let $M$ be a von Neumann algebra on a Hilbert space $\mathscr{H}$ and $\sigma$ an action of $G$ on $M$. Let $\alpha \equiv \pi_{\sigma}, \beta \equiv \hat{\alpha}, \tilde{\alpha} \equiv \hat{\beta}$ and $\tilde{\sigma}$ the action associated with $\tilde{\alpha}$ as in Theorem 2.1. Then $\left(M \otimes_{\alpha} G\right) \otimes_{\beta}^{d} G$ is isomorphic to $M \otimes B\left(L^{2}(G)\right)$ and the isomorphism transforms the action $\tilde{\sigma}$ on the former into the action $\sigma \otimes \operatorname{Ad} \lambda^{\prime}$ on the latter.

Proof. Let $\mathscr{H}_{1} \equiv \mathscr{H} \otimes L^{2}(G)$ and $\mathscr{H}_{2} \equiv \mathscr{H} \otimes L^{2}(G) \otimes L^{2}(G)$. Using (2.9) and (2.10), we set

$$
\Lambda(r) \equiv \lambda_{1}(r) \otimes \lambda(r) \quad \text { and } \quad T_{2}(f) \equiv 1 \otimes 1_{G} \otimes f
$$

for $f$ in $L^{\infty}(G)$. Let $N \equiv M \otimes_{\alpha} G$ and $D \equiv N \otimes_{\beta}^{d} G . \quad N$ is generated by $\alpha(M)$ and $1 \otimes R(G)$ on $\mathscr{H}_{1}$ and $D$ is generated by $\beta(N)$ and $1_{N} \otimes L^{\infty}(G)$ 
on $\mathscr{H}_{2}$. Therefore by (2.9) $D$ is generated by $\alpha(M) \otimes 1_{G}, \Lambda(G)$ and $T_{2}\left(L^{\infty}(G)\right)$. Since

$$
\begin{aligned}
& \left(\Lambda(r) T_{2}(f) \xi\right)(s, t)=\left(T_{2}(f) \xi\right)(s r, t r) \\
& \quad=f(t r) \xi(s r, t r)=f(t r)(\Lambda(r) \xi)(s, t) \\
& \quad=\left(T_{2}(r f) \Lambda(r) \xi\right)(s, t)
\end{aligned}
$$

$\Lambda$ and $T_{2}$ satisfy the commutation relation in the sense of Mackey, [10]. Therefore the von Neumann algebra $B$ generated by $\Lambda(G)$ and $T_{2}\left(L^{\infty}(G)\right)$ is isomorphic to $B\left(L^{2}(G)\right)$, and hence $D$ is isomorphic to $\left(D \cap B^{\prime}\right) \otimes B . \quad$ Put

$$
\pi(x) \equiv \operatorname{Ad} 1 \otimes V^{*}\left(\alpha(x) \otimes 1_{G}\right)
$$

on $M$. Then $\pi$ is an isomorphism of $M$ into $B\left(\mathscr{H} \otimes L^{2}(G) \otimes L^{2}(G)\right)$ and satisfies

$$
(\pi(x) \xi)(s, t)=\sigma_{s t^{-1}}(x) \xi(s, t)
$$

Since

$$
\begin{aligned}
& \left(\pi(x) T_{2}(f) \xi\right)(s, t)=\sigma_{s t^{-1}}(x)\left(T_{2}(f) \xi\right)(s, t) \\
& \quad=\sigma_{s t^{-1}}(x) f(t) \xi(s, t)=f(t) \sigma_{s t^{-1}}(x) \xi(s, t) \\
& \quad=\left(T_{2}(f) \pi(x) \xi\right)(s, t)
\end{aligned}
$$

and

$$
\begin{aligned}
& (\pi(x) \Lambda(r) \xi)(s, t)=\sigma_{s t^{-1}}(x)(\Lambda(r) \xi)(s, t) \\
& \quad=\sigma_{s r(t r)^{-1}}(x) \xi(s r, t r)=(\pi(x) \xi)(s r, t r) \\
& \quad=(\Lambda(r) \pi(x) \xi)(s, t)
\end{aligned}
$$

we have $\pi(M) \subset B^{\prime}$.

Let $K(G \times G, \mathscr{H})$ be the set of all continuous functions on $G \times G$ with compact carriers and with values in $\mathscr{H}$. For each $f$ and $g$ in $K(G)$ with $g \geq 0$ and $\|g\|_{1}=1$ we put

$$
x_{f, g} \equiv \int f(r)\left(\alpha\left(\sigma_{r}(x)\right) \otimes 1_{G}\right) T_{2}\left(g_{r}\right) d r,
$$


where $g_{r}(s) \equiv g(r s)$. Then $x_{f, g} \in D$. For any $\xi$ and $\eta$ in $K(G \times G, \mathscr{H})$ we have

$$
\begin{aligned}
\left(x_{f, g} \xi \mid \eta\right) & =\int f(r)\left(\left(\alpha\left(\sigma_{r}(x)\right) \otimes 1_{G}\right) T_{2}\left(g_{r}\right) \xi \mid \eta\right) d r \\
& =\iiint f(r) g(r t)\left(\sigma_{s r}(x) \xi(s, t) \mid \eta(s, t)\right) d s d t d r \\
& =\iiint f\left(r t^{-1}\right) g(r)\left(\sigma_{s r t^{-1}}(x) \xi(s, t) \mid \eta(s, t)\right) d r d s d t
\end{aligned}
$$

Since $r \mapsto f\left(r t^{-1}\right)\left(\sigma_{s r t^{-1}}(x) \xi(s, t) \mid \eta(s, t)\right)$ belongs to $K(G)$, when the measure $g(r) d r$ converges to the Dirac measure $\varepsilon_{e}$ at the unit $e$ of $G$, the right hand side converges to

$$
\iint f\left(t^{-1}\right)\left(\sigma_{s t^{-1}}(x) \xi(s, t) \mid \eta(s, t)\right) d s d t
$$

and this converges to $(\pi(x) \xi \mid \eta)$ as $f$ converges to the constant 1 function uniformly on each compact subset of $G$. Since $K(G \times G, \mathscr{H})$ is dense in $\mathscr{H}_{2}$, and since $\left\|x_{f, g}\right\| \leq\|f\|\|x\|\|g\|_{1}, x_{f, g}$ converges weakly to $\pi(x)$ and hence $\pi(M) \subset D$, namely, $\pi(M) \subset D \cap B^{\prime}$.

Next we shall show that $D$ is generated by $\pi(M)$ and $B$. For each $f$ and $g$ in $K(G)$ we put

$$
y_{f, g} \equiv \int f(r) \pi\left(\sigma_{r}^{-1}(y)\right) T_{2}\left(g_{r}\right) d r .
$$

Then $y_{f, g} \in(\pi(M) \cup B)^{\prime \prime}$. For each $\xi$ and $\eta$ in $K(G \times G, \mathscr{H})$ we have

$$
\begin{aligned}
\left(y_{f, g} \xi \mid \eta\right) & =\int f(r)\left(\pi\left(\sigma_{r}^{-1}(y)\right) T_{2}\left(g_{r}\right) \xi \mid \eta\right) d r \\
& =\iiint f(r) g(r t)\left(\sigma_{s t^{-1} r^{-1}}(y) \xi(s, t) \mid \eta(s, t)\right) d s d t d r \\
& =\iiint f\left(r t^{-1}\right) g(r)\left(\sigma_{s r^{-1}}(y) \xi(s, t) \mid \eta(s, t)\right) d r d s d t
\end{aligned}
$$

By the same reason as above, when the measure $g(r) d r$ converges to $\varepsilon_{e}$, the right hand side converges to

$$
\iint f\left(t^{-1}\right)\left(\sigma_{s}(y) \xi(s, t) \mid \eta(s, t)\right) d s d t
$$


which converges to $\left(\left(\alpha(y) \otimes 1_{G}\right) \xi \mid \eta\right)$ as $f$ tends to 1 in an appropriate sense. Thus $\alpha(M) \otimes 1_{G} \subset(\pi(M) \cup B)^{\prime \prime}$, and hence $D \subset(\pi(M) \cup B)^{\prime \prime}$. Since the converse inclusion is obtained in the above, $D=(\pi(M) \cup B)^{\prime \prime}$. Therefore $D$ is isomorphic to $\pi(M) \otimes B$.

By Theorem $2.1 \tilde{\alpha}$ and $\tilde{\sigma}$ satisfies

$$
(\tilde{\alpha}(z) \xi)(r)=\tilde{\sigma}_{r}(z) \xi(r) \quad z \in D
$$

for $\xi \in \mathscr{H}_{2} \otimes L^{2}(G)$. Since we know from (2.12) that

$$
\hat{\alpha}(\beta(y))=\beta(y) \otimes 1_{G} \quad \text { and } \quad \tilde{\alpha}\left(T_{2}(f)\right)=1 \otimes 1_{G} \otimes \varepsilon f,
$$

we have

$$
\begin{gathered}
\begin{array}{c}
\tilde{\sigma}_{r}\left(\alpha(x) \otimes 1_{G}\right) \xi(r) \\
=\left(\left(\alpha(x) \otimes 1_{G} \otimes 1_{G}\right) \xi\right)(r) \\
=\left(\alpha(x) \otimes 1_{G}\right) \xi(r)
\end{array} \\
\tilde{\sigma}_{r}(\Lambda(s)) \xi(r)=\left(\left(\Lambda(s) \otimes 1_{G}\right) \xi\right)(r)=\Lambda(s) \xi(r)
\end{gathered}
$$

and

$$
\begin{aligned}
\tilde{\sigma}_{r}\left(T_{2}(f)\right) \xi(r) & =\left(\left(1 \otimes 1_{G} \otimes \varepsilon f\right) \xi\right)(r) \\
& =T_{2}\left(f_{r-1}\right) \xi(r) .
\end{aligned}
$$

Since $\left[\Lambda(r), \lambda_{2}^{\prime}\left(r^{\prime}\right)\right]=0$ for all $r, r^{\prime} \in G$, it follows from (3.4) that

$$
\tilde{\sigma}_{r}=\operatorname{Ad} \lambda_{2}^{\prime}(r)
$$

on $B$.

We apply (3.3) and (3.4) to $x_{f, g}$ defined by (3.2). Then

$$
\begin{aligned}
& \tilde{\sigma}_{a}\left(x_{f, g}\right) \xi(s, t) \\
& \quad=\int f(r)\left(\alpha\left(\sigma_{r}(x)\right) \otimes 1_{G}\right) \tilde{\sigma}_{a}\left(T_{2}\left(g_{r}\right)\right) \xi(s, t) d r \\
& \quad=\int f(r) g\left(r a^{-1} t\right) \sigma_{s r}(x) \xi(s, t) d r \\
& \quad=\int f\left(r t^{-1} a\right) g(r) \sigma_{s r t^{-1}}\left(\sigma_{a}(x)\right) \xi(s, t) d r
\end{aligned}
$$


When $g(r) d r$ converges to $\varepsilon_{e},\left(\tilde{\sigma}_{a}\left(x_{f, g}\right) \xi \mid \eta\right)$ converges to

$$
\begin{aligned}
& \iint f\left(t^{-1} a\right)\left(\sigma_{s t^{-1}}\left(\sigma_{a}(x)\right) \xi(s, t) \mid \eta(s, t)\right) d s d t \\
& \quad=\iint f\left(t^{-1} a\right)\left(\pi\left(\sigma_{a}(x)\right) \xi(s, t) \mid \eta(s, t)\right) d s d t
\end{aligned}
$$

Therefore, if $g(r) d r$ converges to $\varepsilon_{e}$ and then $f$ to 1 , then $x_{f, g}$ converges to $\pi(x)$ as before and hence

$$
\tilde{\sigma}_{a}(\pi(x))=\pi\left(\sigma_{a}(x)\right) \quad x \in M
$$

Combining (3.5) and (3.6), we have

$$
\rho \circ \tilde{\sigma}_{a}=\left(\sigma_{a} \otimes \operatorname{Ad} \lambda_{1}^{\prime}(a)\right) \circ \rho
$$

for all $a \in G$, where $\rho$ is the isomorphism of $D$ onto $M \otimes B$ obtained before.

Q.E.D.

\section{Some Technical Lemmas for $\beta$}

Let $N$ be a von Neumann algebra on a Hilbert space $\mathscr{K}$ and $\beta$ a dual action of $G$ on $N$. For any $\phi$ in $R(G)_{*}$ and $\omega$ in $N_{*}$ we define linear mappings $\beta_{\phi}$ on $N$ and $\Phi_{\omega}$ of $N$ into $R(G)$ by

$$
\begin{aligned}
& <\beta_{\phi}(x), \omega^{\prime}>=<\beta(x), \omega^{\prime} \otimes \phi> \\
& <\Phi_{\omega}(x), \phi^{\prime}>=<\beta(x), \omega \otimes \phi^{\prime}>
\end{aligned}
$$

for all $x \in N, \omega^{\prime} \in N_{*}$ and $\phi^{\prime} \in R(G)_{*}$. Let $\beta_{*}$ denote the mapping of $(N \otimes R(G))_{*}$ onto $N_{*}$ defined by

$$
<x, \beta_{*}(\omega \otimes \phi)>=<\beta(x), \omega \otimes \phi>
$$

for all $\omega \in N_{*}$ and $\phi \in R(G)_{*}$.

Since $\gamma$ is a dual action of $G$ on $R(G), \gamma_{\phi}$ and $\gamma_{*}$ are defined by (4.1) and (4.2).

Lemma 4.1. Let $\phi$ and $\psi$ be elements in $R(G)_{*}$.

(i) $\beta_{\phi \psi}=\beta_{\phi} \beta_{\psi}$. 
(ii) $\gamma_{\psi}\left(\Phi_{\omega}(x)\right)=\Phi_{\omega}\left(\beta_{\psi}(x)\right)$ for $\omega \in N_{*}$ and $x \in N$.

(iii) $\left\langle\beta\left(\beta_{\psi}(x)\right), \omega \otimes \phi>=\left\langle\gamma \Phi_{\omega}(x), \psi \otimes \phi>\right.\right.$.

Proof. (i) If $\omega \in N_{*}$ and $x \in N$, then

$$
\begin{aligned}
& <\beta_{\phi \psi}(x), \omega>=<\beta(x), \omega \otimes \phi \psi> \\
& =<(\iota \otimes \gamma) \beta(x), \omega \otimes \phi \otimes \psi> \\
& =<(\beta \otimes \iota) \beta(x), \omega \otimes \phi \otimes \psi> \\
& =<\beta(x), \beta_{*}(\omega \otimes \phi) \otimes \psi> \\
& =<\beta_{\psi}(x), \beta_{*}(\omega \otimes \phi)> \\
& =<\beta\left(\beta_{\psi}(x)\right), \omega \otimes \phi> \\
& =<\beta_{\phi}\left(\beta_{\psi}(x)\right), \omega>.
\end{aligned}
$$

(ii) If $\phi \in R(G)_{*}$, then

$$
\begin{aligned}
& <\gamma_{\psi} \Phi_{\omega}(x), \phi>=<\gamma \Phi_{\omega}(x), \phi \otimes \psi> \\
& =<\Phi_{\omega}(x), \phi \psi>=<\beta(x), \omega \otimes \phi \psi> \\
& =<\beta\left(\beta_{\psi}(x)\right), \omega \otimes \phi> \\
& =<\Phi_{\omega}\left(\beta_{\psi}(x)\right), \phi>.
\end{aligned}
$$

(iii) From (4.4) we have

$$
\begin{aligned}
& <\beta\left(\beta_{\psi}(x)\right), \omega \otimes \phi>=<\Phi_{\omega}(x), \psi \phi> \\
& =<\gamma \Phi_{\omega}(x), \psi \otimes \phi>.
\end{aligned}
$$

Q.E.D.

The following lemma is an immediate consequence of $[11$, Theorem 5, Chapter 3]. For the sake of completeness we shall give a direct proof.

Lemma 4.2. Let $L$ be $a$ von Neumann algebra and $t \mapsto u(t) a$ weakly continuous unitary representation of $G$ in $L$. If $\phi$ is an ele- 
ment of $L_{*}$, then $t \mapsto u(t)^{*} \phi\left(\right.$ or $\left.u(t) \phi, \phi u(t), \phi u(t)^{*}\right)$ is continuous (in norm).

By means of this lemma we know that the functions in the above lemma are Bochner integrable on every compact subset of $G$ and their integrals exist in $L_{*}$.

Proof. For each $f$ in $L^{1}(G)$ we denote by $u(f)$ the integral

$$
\int f(t) u(t) d t
$$

Let $L_{0}$ be the set of all $u(f)^{*} \psi$ with $f \in K(G)$ and $\psi \in L_{*}$. Since $u(f)^{*} \psi$ converges weakly to $\psi$ as $f(t) d t$ tends to $\varepsilon_{e}, L_{0}$ is weakly dense in $L_{*}$ and hence it is total in $L_{*}$ in norm by the Hahn-Banach's separation theorem.

For any $\phi$ in $L_{0}$ of the form $u(f)^{*} \psi$ we have

$$
\begin{aligned}
\left\|\left(u(t)^{*}-u(s)^{*}\right) \phi\right\| & \leqq\|u(f)(u(t)-u(s))\|\|\psi\| \\
& \leqq\left\|_{t^{-1}} f-_{s^{-1}} f\right\|_{1}\|\psi\| .
\end{aligned}
$$

Since $L_{0}$ is total in $L_{*}$ in norm, $t \mapsto u(t)^{*} \phi$ is continuous for all $\phi$ in $L_{*}$.

As for the remaining functions $t \mapsto u(t) \phi, \phi u(t)$ and $\phi u(t)^{*}$ we can give their proofs in a similar way. $\quad$ Q.E.D.

As $<\lambda(s), \lambda(r)^{*} \phi>=<\lambda\left(s r^{-1}\right), \phi>$, we have

$$
\lambda_{*}\left(\lambda(r)^{*} \phi\right)=\operatorname{Ad} \lambda(r)^{*}\left(\lambda_{*} \phi\right) .
$$

The following two lemmas are crucial from the technical point of view. In particular, Lemma 4.3 plays a role of Fourier expansion.

Lemma 4.3. Let $\phi$ be elements in $R(G)_{*}$ satisfying $\lambda_{*} \phi \in K(G)_{+}$, $\left\|\Delta \lambda_{*} \phi\right\|_{1}=1$ and $\left\langle\lambda(r)^{*}, \phi>d r\right.$ tends to $\varepsilon_{e}$.

(i) If $\psi$ is an element in $R(G)_{*}$ with $\lambda_{*} \psi \in L^{1}(G)$, the integral

$$
\int<\lambda(r), \psi>\lambda(r)^{*} \phi d r
$$


exists in $R(G)_{*}$, is bounded by $\|\psi\|$ and converges weakly to $\psi$.

(ii) If $x=\beta_{\rho}(x)$ for some $\lambda_{*} \rho \in K(G)$, the integral

$$
\int \beta_{\lambda(r)^{*} \phi}(x) \otimes \lambda(r) d r
$$

exists in $N \otimes_{\gamma^{*}} R(G)$, is bounded by $\|x\|$ and converges weakly* to $\beta(x)$, where $\gamma^{*}$ is the dual norm of $\gamma$-norm.

Proof. (i) We denote (4.5) by $\psi_{\phi}$. By Lemma 4.2, $\quad r \mapsto \lambda(r)^{*} \phi$ is continuous. Since $\lambda_{*} \psi \in L^{1}(G)$ by assumption and $\left\|\lambda(r)^{*} \phi\right\|=\|\phi\|$, the function $r \mapsto\left\langle\lambda(r), \psi>\left(\lambda(r)^{*} \phi\right)\right.$ is Bochner integrable and hence $\psi_{\phi}$ exists in $R(G)_{*}$. If $f \in L^{1}(G)$, then

$$
\begin{aligned}
& \int<\lambda(r)^{*}, \phi><\lambda(r) \lambda(f), \psi>d r \\
& =\int<\lambda(r)^{*}, \phi>\int f(s)<\lambda(r s), \psi>d s d r \\
& =\iint<\lambda\left(s r^{-1}\right), \phi>f(s) d s<\lambda(r), \psi>d r \\
& =\int<\lambda(f), \lambda(r)^{*} \phi><\lambda(r), \psi>d r \\
& =<\lambda(f), \psi_{\phi}>
\end{aligned}
$$

The integral on the left hand side

$$
\int<\lambda(r)^{*}, \phi>(\psi \lambda(r)) d r
$$

exists in $R(G)_{*}$ by a similar reason as above and hence coincides with $\psi_{\phi}$ by (4.7). Therefore

$$
<x, \psi_{\phi}>=\int<\lambda(r)^{*}, \phi><\lambda(r) x, \psi>d r
$$

for all $x \in R(G)$. Here, since $r \mapsto\langle\lambda(r) x, \psi\rangle$ is continuous, if $\left\langle\lambda(r)^{*}\right.$, $\phi>d r$ converges to $\varepsilon_{e}$, then $\psi_{\phi}$ converges weakly to $\psi$.

From (4.8) and the assumption $\left\|\Delta \lambda_{*} \phi\right\|_{1}=1$ it follows that the norm of $\psi_{\phi}$ is majorized by $\|\psi\|$. 
(ii) Since $\operatorname{car} \lambda_{*}\left(\left(\lambda(r)^{*} \phi\right) \rho\right) \subset\left(\operatorname{car} \lambda_{*} \phi\right) r \cap \operatorname{car} \lambda_{*} \rho, \quad r \mapsto \beta_{\lambda(r)^{*} \phi}(x)=\beta_{\left(\lambda(r)^{*} \phi\right) \rho}$ $(x)$ has a compact carrier. Since $r \mapsto \beta_{\lambda(r)^{*} \phi}$ is continuous and $\left\|\beta_{\lambda(r)^{*} \phi}(x)\right\|$ $\leq\|\phi\|\|x\|, r \mapsto \beta_{\lambda(r)^{*} \phi}(x)$ is Bochner integrable and hence (4.6) exists in $N \otimes R(G)$. If $\omega \in N_{*}$ and $\psi \in R(G)_{*}$ with $\lambda_{*} \psi \in K(G)$, then

$$
\begin{aligned}
& <\int \beta_{\lambda(r)^{*} \phi}(x) \otimes \lambda(r) d r, \omega \otimes \psi> \\
& \quad=\int<\beta_{\lambda(r)^{*} \phi}(x), \omega><\lambda(r), \psi>d r \\
& =\int<\beta(x), \omega \otimes \lambda(r)^{*} \phi><\lambda(r), \psi>d r,
\end{aligned}
$$

which converges to $\langle\beta(x), \omega \otimes \psi\rangle$ by (i). Since the set of all $\psi \in R(G)_{*}$ satisfying $\lambda_{*} \psi \in K(G)$ is weakly dense, it is dense in $R(G)_{*}$. Since the absolute value of the right hand side of (4.9) is majorized by $\|x\|\|\omega\|\|\psi\|$ by (i), $\gamma^{*}$-norm of (4.6) is bounded by $\|x\|$. Therefore (4.6) converges weakly* to $\beta(x)$.

Q.E.D.

The above (ii) in Lemma 4.3 or the following remark can be used to prove (ii) in Theorem 7.1. However, we shall intend to utilize the former in this paper.

Remark. If $x$ is of the form $\beta_{\psi}(y)$ for some $\psi \in R(G)_{*}$ with $\lambda_{*} \psi$ $\in K(G)$, then (4.6) exists in $N \otimes R(G)$ and converges $\sigma$-weakly to $\beta(x)$. For this it suffices to show that (4.6) is uniformly bounded in $\phi$. If we use the argument which will be done in Lemma 7.3, the integrals

$$
\begin{aligned}
F_{\phi, \psi}^{0} & \equiv \int\left(\lambda(r)^{*} \phi\right) \psi \otimes\left(\left(1_{G} \otimes \lambda(r)\right) \omega^{\prime}\right) d r \\
G_{\phi}^{0} & \equiv \int\left\langle\lambda(r)^{*}, \phi>\left(\omega^{\prime}\left(1_{G} \otimes \lambda(r)\right)\right) \circ A d W^{*} d r\right.
\end{aligned}
$$

exist as vector forms and satisfy

$$
<\gamma \Phi_{\omega}(y) \otimes 1_{G}, F_{\phi, \psi}^{0}>=<\beta\left(\beta_{\psi}(y)\right) \otimes 1_{G}, \omega \otimes G_{\phi}^{0}>
$$

Since 


$$
\begin{aligned}
& <(\beta \otimes \iota)\left(\int \beta_{\left(\lambda(r)^{*} \phi\right)}(x) \otimes \lambda(r) d r\right), \omega \otimes \omega^{\prime}> \\
& \quad=<\gamma \Phi_{\omega}(y) \otimes 1_{G}, F_{\phi, \psi}^{0}> \\
& \quad=<\left(\int<\lambda(r)^{*}, \phi>1 \otimes 1_{G} \otimes \lambda(r) d r\right) A d 1 \otimes W^{*}\left(\beta(x) \otimes 1_{G}\right), \omega \otimes \omega^{\prime}>,
\end{aligned}
$$

it follows from the right hand side that (4.6) is bounded by $\|x\|$ under the assumption of Lemma 4.3.

Lemma 4.4. Let $\phi$ be an element of $R(G)_{*}$ satisfying $\lambda_{*} \phi \in K(G)_{+}$ and $\left\|\Delta \lambda_{*} \phi\right\|_{1}=1$.

(i) If $\psi$ is an element in $R(G)_{*}$ with $\lambda_{*} \psi \in K(G)$, the integral

$$
\int\left(\lambda(r)^{*} \phi\right) \psi d r
$$

exists in $R(G)_{*}$ and coincides with $\psi$.

(ii) If $x=\beta_{\rho}(x)$ for some $\lambda_{*} \rho \in K(G)$, the integral

$$
\int \beta_{\lambda(r)^{*} \phi}(x) d r
$$

exists in $N$ and coincides with $x$.

Proof. (i) Since $\lambda_{*} \phi, \lambda_{*} \psi \in K(G)$ and $\operatorname{car} \lambda_{*}\left(\left(\lambda(r)^{*} \phi\right) \psi\right) \subset\left(\operatorname{car} \lambda_{*} \phi\right) r$ $\cap \operatorname{car} \lambda_{*} \psi, r \mapsto\left(\lambda(r)^{*} \phi\right) \psi$ has a compact carrier. Since $\left\|\left(\lambda(r)^{*} \phi\right) \psi\right\| \leq$ $\|\phi\|\|\psi\|$ and $r \mapsto\left(\lambda(r)^{*} \phi\right) \psi$ is continuous by Lemma 4.2, (4.10) exists in $R(G)_{*}$. Since

$$
\int<\lambda(s), \lambda(r)^{*} \phi>d r=\int<\lambda(r)^{*}, \phi>d r=1
$$

for all $s \in G$ by assumption, we have for any $f$ in $L^{1}(G)$

$$
\begin{aligned}
<\lambda(f), \psi> & =\int<\lambda(r)^{*}, \phi>d r<\lambda(f), \psi> \\
& =\iint f(s)<\gamma \lambda(s), \lambda(r)^{*} \phi \otimes \psi>d r d s \\
& =<\lambda(f), \int\left(\lambda(r)^{*} \phi\right) \psi d r>
\end{aligned}
$$


Since $\lambda\left(L^{1}(G)\right)$ is $\sigma$-weakly dense in $R(G), \psi$ is given by (4.10).

(ii) By a similar reason as in the proof of (ii) in Lemma 4.3, (4.11) exists in $N$ and is bounded. For any $\omega \in N_{*}$ and any $\psi \in R(G)_{*}$ with $\lambda_{*} \psi \in K(G)$

$$
\begin{aligned}
& <\beta(x), \omega \otimes \psi>=\int<\beta(x), \omega \otimes\left(\lambda(r)^{*} \phi\right) \psi>d r \\
& =<\beta\left(\int \beta_{\lambda(r)^{*} \phi}(x) d r\right), \omega \otimes \psi>. \quad \text { (By (4.3)). }
\end{aligned}
$$

Since the set of $\psi \in R(G)_{*}$ with $\lambda_{*} \psi \in K(G)$ is dense in $R(G)_{*}$, the linear span of $\omega \otimes \psi$ with $\lambda_{*} \psi \in K(G)$ is dense in $(N \otimes R(G))_{*}$. Therefore (4.11) coincides with $x$.

Q.E.D.

Lemma 4.5. If $x \in N$, then $x$ belongs to the von Neumann algebra generated by $\beta_{\psi}(x)$ with $\lambda_{*} \psi \in K(G)$.

Proof. Let $N_{0}$ be the von Ncumann algebra generated by $\beta_{\psi}(x)$ with $\lambda^{*} \psi \in K(G)$. If $\omega \in N_{*}$ annihilates on $N_{0}$, then

$$
<\beta(x), \omega \otimes \psi>=<\beta_{\psi}(x), \omega>=0
$$

for all $\psi$ with $\lambda_{*} \psi \in K(G)$. Since the set of all $\psi$ with $\lambda_{*} \psi \in K(G)$ is dense in $R(G)_{*}$, (4.13) holds for all $\psi \in R(G)_{*}$. Therefore $\beta(x)$ belongs to $N_{0} \otimes R(G)$ by [19]. By considering $\beta_{\psi \prime}(x)$ as $x$ in (4.13), $\beta \uparrow N_{0}$ is a dual action of $G$ on $N_{0}$ and hence $x \in N_{0}$ by [22, Proposition II. 1.1].

Q.E.D.

\section{Spectrum of $\beta$}

The spectrum of an action of $G$ on a $C^{*}$-algebra was investigated by the method of abstract harmonic analysis, [2]. We shall define the corresponding concept for a dual action of $G$ on $N$ by using the same ideas. When the set of $x$ in $N$ is trivial whose spectrum with respect to $\beta$ is $\{e\}, \beta$ is considered to be ergodic.

The basic theorem in Gelfand's theory for a commutative Banach algebra tells us that there is a bijection between the set of all maximal regular ideals $m$ of $R(G)_{*}$ and the spectrum $G$ satisfying 


$$
m^{\perp}=\boldsymbol{C} \lambda(t) \quad \text { and } \quad\{\lambda(t)\}^{\perp}=m
$$

for $t \in G$. The Tauberian theorem is generalized by Eymard [5] as the following: if $m$ is a closed ideal of $R(G)_{*}$ such that for any $t \in G$ there exists a $\phi \in m$ with $\left\langle\lambda(t), \phi>\neq 0\right.$, then $m=R(G)_{*}$. Therefore every proper closed ideal is included in a maximal regular ideal.

Definition 5.1. For any $\phi$ in $R(G)_{*}$ let $\Gamma(\phi)$ denote the set of all $t \in G$ with $\langle\lambda(t), \phi\rangle=0$. Let

$$
\begin{gathered}
\operatorname{sp}(\beta) \equiv \cap\left\{\Gamma(\phi): \beta_{\phi}=0\right\} \\
\left(\text { resp. } \operatorname{sp}_{\beta}(x) \equiv \cap\left\{\Gamma(\phi): \beta_{\phi}(x)=0\right\} \text { for } x \in N\right) .
\end{gathered}
$$

Let $m_{\beta}$ (resp. $m_{x}$ ) denote the set of all $\phi \in R(G)_{*}$ with $\beta_{\phi}=0$ (resp. $\left.\beta_{\phi}(x)=0\right)$.

From (i) in Lemma 4.1 it follows that $m_{\beta}$ and $m_{x}$ are closed ideals of $R(G)_{*}$ and $\operatorname{sp}(\beta)\left(\right.$ resp. $\left.\mathrm{sp}_{\beta}(x)\right)$ is the hull of $m_{\beta}$ (resp. $\left.m_{x}\right)$. Besides, if $\operatorname{sp}_{\beta}(x)=\phi$, then $m_{x}=R(G)_{*}$ by Tauberian theorem and hence $x=0$.

Proposition 5.2. For a non zero $x \in N$ and $t \in G$ the following four conditions are equivalent:

(i) $\operatorname{sp}_{\beta}(x)=\{t\}$;

(ii) $\beta(x)=x \otimes \lambda(t)$;

(iii) $\beta_{\phi}(x)=<\lambda(t), \phi>x$ for all $\phi \in R(G)_{*}$; and

(iv) $\Phi_{\omega}(x)=<x, \omega>\lambda(t)$ for all $\omega \in N_{*}$.

Proof. The equivalence among conditions (ii), (iii) and (iv) is immediate from (4.1). It suffices to show the implications (iii) $\Rightarrow$ (i) and (i) $\Rightarrow$ (ii).

(iii) $\Rightarrow$ (i) Suppose the condition (iii). Since $\beta_{\phi}(x)=0$ implies $<\lambda(t)$, $\phi>=0$ for all $\phi \in R(G)_{*}$, we have $t \in \operatorname{sp}_{\beta}(x)$. For any $s \in G$ with $s \neq t$ we can select a $\psi$ in $R(G)_{*}$ satisfying $\langle\lambda(t), \psi\rangle=0$ and $\langle\lambda(s), \psi\rangle \neq 0$. Since $\langle\lambda(t), \psi\rangle=0$ implies $\beta_{\psi}(x)=0$ by assumption, $s$ does not belong to $\operatorname{sp}_{\beta}(x)$. Since $s$ is arbitrary with $s \neq t$, the condition (i) is obtained.

(i) $\Rightarrow$ (ii) Suppose the condition (i). Let $m_{t}$ denote the maximal regular ideal associated with $t \in G$ by (5.1). Since $m_{x}$ is included in 
$m_{t}$ but not in $m_{s}$ with $s \neq t, m_{x}$ is primary. By using [5, (4.10)], $m_{x}=$ $m_{t}$ and hence

$$
<\Phi_{\omega}(x), \phi>=<\beta_{\phi}(x), \omega>=0
$$

for all $\phi \in m_{t}$. Since $m_{t}$ is a maximal regular ideal, $\Phi_{\omega}(x)=\mu(\omega) \lambda(t)$ for some $\mu(\omega) \in \boldsymbol{C}$. From the linearity of $\Phi_{\omega}$ in $\omega \in N_{*}$, and $\left\|\Phi_{\omega}(x)\right\|$ $\leq\|x\|\|\omega\|$ it follows that $\mu$ is a bounded linear form on $N_{*}$, namely, we have a $y$ in $N$ with $\Phi_{\omega}(x)=<y, \omega>\lambda(t)$. Since for any $\omega$ in $N_{*}$ and any $\phi$ in $R(G)_{*}$

$$
\begin{aligned}
& <\beta(x), \omega \otimes \phi>=<\Phi_{\omega}(x), \phi> \\
& =<y, \omega><\lambda(t), \phi>=<y \otimes \lambda(t), \omega \otimes \phi>,
\end{aligned}
$$

we have $\beta(x)=y \otimes \lambda(t)$. Since

$$
\begin{aligned}
& \beta(y) \otimes \lambda(t)=(\beta \otimes \iota)(y \otimes \lambda(t))=(\beta \otimes \iota) \beta(x) \\
& =(\iota \otimes \gamma) \beta(x)=(\iota \otimes \gamma)(y \otimes \lambda(t))=y \otimes \lambda(t) \otimes \lambda(t),
\end{aligned}
$$

we have $(\beta(y)-y \otimes \lambda(t)) \otimes \lambda(t)=0$ and hence $\beta(y)=y \otimes \lambda(t)=\beta(x)$. Therefore $x=y$ and hence $\beta(x)=x \otimes \lambda(t)$.

Definition 5.3. Let $N^{\beta}$ denote the set of all $x$ in $N$ with $\operatorname{sp}_{\beta}(x)$ $=\{e\}$, and $M^{\alpha}$ the fixed point algebra of $\alpha_{t}$ for all $t \in G$.

The following proposition is not necessary for later use. For each element $y \in R(G)$ the carrier $\operatorname{supp}(y)$ of $y$ is defined by Eymard [5] as $\operatorname{sp}_{\gamma}(y)$. We can describe $\operatorname{sp}_{\beta}(x)$ in terms of the carriers of $\Phi_{\omega}(x), \omega \in N_{*}$.

Proposition 5.4. $\operatorname{sp}_{\beta}(x)$ is the closure of the union of $\operatorname{sp}_{\gamma}\left(\Phi_{\omega}(x)\right)$ for all $\omega$ in $N_{*}$.

Proof. If $\omega \in N_{*}$ and $\psi \in R(G)_{*}$, then

$$
<\gamma_{\phi}\left(\Phi_{\omega}(x)\right), \psi>=<\beta\left(\beta_{\phi}(x)\right), \omega \otimes \psi>
$$

by (iii) in Lemma 4.1. Hence $\beta_{\phi}(x)=0$ if and only if $\gamma_{\phi} \Phi_{\omega}(x)=0$ for all $\omega$ in $N_{*}$. Therefore 


$$
m_{x}=\bigcap_{\omega \in N_{*}}\left\{\phi \in R(G)_{*}: \gamma_{\phi} \Phi_{\omega}(x)=0\right\}
$$

whose hull gives the desired result.

Q.E.D.

\section{Fixed Points of $\alpha$ and $\beta$}

Let $\alpha$ be an action of $G$ on $M$ and $\hat{\alpha}$ the dual action of $G$ on $M \otimes_{\alpha} G$ dual to $\alpha$. A generalized conditional expectation of $M \otimes_{\alpha} G$ onto $\left(M \otimes_{\alpha} G\right)^{\hat{\alpha}}$ has been investigated by Landstad in his forthcomming paper, [9]. Using his results, we shall show that $\left(M \otimes_{\alpha} G\right)^{\hat{\alpha}}=\alpha(M)$. Similar argument for a dual action $\beta$ of $G$ on $N$ has been developed by Haagerup, [6]. In the latter half of this section we shall give an independent argument in order to show $\left(N \otimes_{\beta}^{d} G\right)^{\hat{\beta}}=\beta(N)$.

The case of $\alpha$.

$K(G)$ is a left Hilbert algebra with respect to a product $(f, g) \mapsto$ $f * g$, an involution $f \mapsto \tilde{f}$ and an inner product $(f \mid g) \equiv I(f \bar{g})$. Using the left representation $\pi$ of $K(G)$ we have

$$
(\pi(f) g)(t)=(f * g)(i)=\left(\lambda^{\prime}\left(\Delta^{1 / 2} f\right) g\right)(t) .
$$

The modular conjugation $J$ of $K(G)$ is of the form $(J f)(t)=\Delta(t)^{1 / 2} \tilde{f}(t)$ and $\lambda^{\prime}(t)=J \lambda(t) J$. Therefore $R(G)^{\prime}$ is generated by $\lambda^{\prime}(G)$ and it is the left von Neumann algebra of $K(G)$ by (6.1). The extension $\dot{\psi}$ over $\mathrm{m}_{\psi}$ of the canonical weight $\psi$ on $R(G)_{+}^{\prime}$ associated with $K(G)$ is given by

$$
\dot{\psi}(\pi(\tilde{g} * f))=\psi(\pi(g) * \pi(f))=(f \mid g)=(\tilde{g} * f)(e),
$$

where $e$ denotes the unit of $G$. We denote by $\omega_{e}$ the weight on $R(G)$ defined by $\dot{\psi} \circ \operatorname{Ad} J$. Then

$$
\omega_{e}\left(\lambda\left(\Delta^{1 / 2}(\tilde{g} * f)\right)\right)=(\tilde{g} * f)(e) .
$$

Let $F$ be a net in $R(G)_{*}^{+}$such that $\lambda_{*} F \subset K(G)$ and

$$
\omega_{c}(x)=\sup \{<x, \phi>: \phi \in F\} \quad x \in R(G)_{+} .
$$

Let $\beta$ be a dual action of $G$ on $N$. If $y \in N_{+}$, then $\left\{\beta_{\phi}(y): \phi \in F\right\}$ is an increasing net in $N_{+}$. Define a generalized conditional expectation 
$E_{\beta}$ for $y \in N_{+}$by

$$
E_{\beta}(y) \equiv \sup \left\{\beta_{\phi}(y): \phi \in F\right\} .
$$

Since $\omega \otimes \omega_{e}$ for $\omega \in N_{*}^{+}$is a semi-finite normal weight on $N \otimes R(G)$, we have

$$
<E_{\beta}(y), \omega>=\sup _{\phi}<\beta_{\phi}(y), \omega>=<\beta(y), \omega \otimes \omega_{e}>
$$

for $y \in N_{+}$. Let $\mathfrak{n}_{\beta}$ be the set of all $x \in N$ such that

$$
<\beta\left(x^{*} x\right), \omega \otimes \omega_{e}>\leq \mu_{x}\|\omega\| \quad \omega \in N_{*}^{+}
$$

for some $\mu_{x}>0$. Since $x^{*} y^{*} y x \leq\|y\|^{2} x^{*} x, \mathfrak{n}_{\beta}$ is a left ideal of $N$. Let $\mathfrak{m}_{\beta}=\mathfrak{n}_{\beta}^{*} \mathfrak{n}_{\beta}$ and $\dot{E}_{\beta}$ be the linear extension of $E_{\beta}$ over $\mathfrak{m}_{\beta}$. Then $E_{\beta}$ satisfies

(i) $E_{\beta}(x+y)=E_{\beta}(x)+E_{\beta}(y) \quad x, y \in N_{+}$

(ii) $E_{\beta}(\mu x)=\mu E_{\beta}(x) \quad \mu \geq 0$

(iii) $x_{\iota} \uparrow x$ implies $E_{\beta}\left(x_{\iota}\right) \uparrow E_{\beta}(x) \quad x_{\iota} \in N_{+}$

(iv) $\beta\left(\dot{E}_{\beta}(z)\right)=\dot{E}_{\beta}(z) \otimes 1_{G} \quad z \in \mathfrak{m}_{\beta}$

(v) $\dot{E}_{\beta}\left(b^{*} z b\right)=b^{*} \dot{E}_{\beta}(z) b \quad b \in N_{\beta}$,

where $N_{\beta}$ denotes the set of all $x \in N$ with $\beta(x)=x \otimes 1_{G}$. For instance, (iv) is proved as follows: If $z \in \mathrm{m}_{\beta}^{+}, \omega \in N_{*}^{+}$and $\psi \in R(G)_{*}^{+}$, then

$$
\begin{gathered}
<\beta\left(E_{\beta}(z)\right), \omega \otimes \psi>=\sup _{\phi}<\beta\left(\beta_{\phi}(z)\right), \omega \otimes \psi> \\
=\sup _{\phi}<(\iota \otimes \gamma) \beta(z), \omega \otimes \phi \otimes \psi>
\end{gathered}
$$

According to the choice of $F$ in (6.2) we may assume that $<\lambda(t), \phi>d t$ converges to $\varepsilon_{e}$. Since

$$
\begin{aligned}
& <(\iota \otimes \gamma)(x \otimes \lambda(f)), \omega \otimes \phi \otimes \psi> \\
& \quad=<x, \omega>\int f(t)<\lambda(t), \phi><\lambda(t), \psi>d t,
\end{aligned}
$$

the right hand side converges to

$$
<x, \omega>f(e)<1_{G}, \psi>=<x \otimes \lambda(f), \omega \otimes \omega_{e}><1_{G}, \psi>\text {. }
$$


Now, let $\alpha$ be an action of $G$ on $M, N \equiv M \otimes_{\alpha} G$ and $\beta \equiv \hat{\alpha}$. Using results of Landstad [9, Lemma 2.8 and Corollary 1.3], we know that

(a) if $f \in A(G)$, then $1_{M} \otimes \lambda(f) \in \mathrm{m}_{\beta}$ and $\dot{E}_{\beta}\left(1_{M} \otimes \lambda(f)\right)=<\lambda(f)$, $\omega_{e}>1_{N}$

(b) $N_{\beta} \mathfrak{m}_{\beta} N_{\beta} \subset \mathfrak{m}_{\beta}$

(c) $\dot{E}_{\beta}\left(\mathrm{m}_{\beta}\right)=N_{\beta}$

(d) the mapping $y \in \mathrm{m}_{\beta} \mapsto \dot{E}_{\beta}\left(b^{*} y b\right)$ is $\sigma$-weakly continuous for each $b \in \mathfrak{n}_{\beta}$.

For example, (a) is shown by

$$
\begin{aligned}
< & \beta\left(1_{M} \otimes \lambda(f)\right), \omega \otimes \psi \otimes \omega_{e}> \\
= & \int f(t)<1_{M} \otimes \gamma \lambda(t), \omega \otimes \psi \otimes \omega_{e}>d t \\
& =f(e)<1_{M} \otimes 1_{G}, \omega \otimes \psi> \\
& =<<\lambda(f), \omega_{e}>1_{N}, \omega \otimes \psi>
\end{aligned}
$$

for any $f \in A(G)$. Using these results we have the following proposition.

Proposition 6.1. If $\alpha$ is an action of $G$ on $M$, then $\alpha(M)=(M$ $\left.\otimes_{\alpha} G\right)^{\hat{\alpha}}$.

Proof. Let $N \equiv M \otimes_{\alpha} G$ and $\beta \equiv \hat{\alpha}$. By virtue of Proposition 5.2, $N^{\beta}=N_{\beta}$. Since $\alpha(M) \subset N_{\beta}$ by (2.9), we have only to show the converse inclusion.

Let $N_{0}$ be the set of all

$$
\int\left(1_{M} \otimes \lambda(t)\right) \alpha(x(t)) d t
$$

with $t \mapsto x(t)$ in $K(G, M)$. The linear span $N_{1}$ of all $y^{*} x$ with $x, y \in N_{0}$ is $\sigma$-weakly dense in $N$. Since the convex cone $N_{1}^{+}$spanned by $x^{*} x$ with $x \in N_{0}$ generates linearly $N_{1}, N_{1}^{+}$is $\sigma$-weakly dense in $N_{+}$. Since $N_{1} \subset \mathfrak{m}_{\beta}$ by (a) and (b), $N_{1}^{+}$is $\sigma$-weakly dense in $\mathfrak{m}_{\beta}^{+}$. It follows from (d) that $E_{\beta}\left(\left(1_{M} \otimes \lambda(g)^{*}\right) N_{1}^{+}\left(1_{M} \otimes \lambda(g)\right)\right)$ is $\sigma$-weakly dense in $E_{\beta}\left(\left(1_{M} \otimes \lambda(g)^{*}\right)\right.$ $\left.\mathrm{m}_{\beta}^{+}\left(1_{M} \otimes \lambda(g)\right)\right)$ for all $g \in K(g)$. Since $E_{\beta}$ is normal by (iii)

$$
\underset{g \in K(G)}{\cup} E_{\beta}\left(\left(1_{M} \otimes \lambda(g)^{*}\right) \mathrm{m}_{\beta}^{+}\left(1_{M} \otimes \lambda(g)\right)\right)
$$


is $\sigma$-weakly dense in $E_{\beta}\left(\mathrm{m}_{\beta}^{+}\right)=N_{\beta}^{+}$(by (c)). Consequently,

$$
\bigcup_{g \in K(G)} E_{\beta}\left(\left(1_{M} \otimes \lambda(g)^{*}\right) N_{\mathrm{j}}^{+}\left(1_{M} \otimes \lambda(g)\right)\right)
$$

is $\sigma$-weakly dense in $N_{\beta}^{+}$. Since $E_{\beta}\left(\left(1_{M} \otimes \lambda(g)^{*}\right) N_{1}^{+}\left(1_{M} \otimes \lambda(g)\right)\right) \subset \alpha(M)$ by (v) and (a), we have $N_{\beta}^{+} \subset \alpha(M)$ and hence $N_{\beta} \subset \alpha(M)$ Q. E. D.

The case of $\beta$.

Let's recall $\alpha_{g}$ defined by (2.4). Let $I^{\prime}$ be the left invariant Haar integral or $I^{\prime}(f) \equiv I(\Delta f)$. Since $I^{\prime}$ is a semi-finite faithful normal weight on $L^{\infty}(G)$, there exists an increasing net $F \subset K(G)_{+}$such that

$$
I^{\prime}(f)=\sup \{I(f g): g \in F\}, \quad f \in L^{\infty}(G)_{+} \cdot
$$

If $y \in M_{+}$, then $\left\{\alpha_{g}(y): g \in F\right\}$ is an increasing net in $M_{+}$. Define $E_{\alpha}$ for $y \in M_{+}$by

$$
E_{\alpha}(y) \equiv \sup \left\{\alpha_{g}(y): g \in F\right\} .
$$

Since $\omega \otimes I^{\prime}$ for $\omega \in M_{*}^{+}$is a semi-finite normal weight on $M \otimes L^{\infty}(G)$, we have

$$
<E_{\alpha}(y), \omega>=\sup _{g}\langle\alpha(y), \omega \otimes g>=\langle\alpha(y), \omega \otimes \Delta>
$$

Since $E_{\alpha}(y)$ is not necessarily bounded, we shall take out the bounded part by considering the set $n_{\alpha}$ of all $x \in M$ such that

$$
<\alpha\left(x^{*} x\right), \omega \otimes \Delta>\leq \mu_{x}\|\omega\| \quad \omega \in M_{*}^{+}
$$

for some $\mu_{x}>0$. Since $x^{*} y^{*} y x \leq\|y\|^{2} x^{*} x, \mathfrak{n}_{\alpha}$ is a left ideal of $M$. Put $\mathrm{m}_{\alpha} \equiv \mathrm{n}_{\alpha}^{*} \mathrm{n}_{\alpha} . \quad E_{\alpha}$ is, by (6.4), linear and normal on $M_{+}$and is extended canonically over $m_{\alpha}$, which is denoted by $\dot{E}_{\alpha}$.

Lemma 6.2. Let $\alpha$ be an action of $G$ on $M$ and let $M_{\alpha}$ be the set of all $x \in M$ with $\alpha(x)=x \otimes 1$. If $E_{\alpha}$ and $\mathrm{m}_{\alpha}$ are defined as above, then
(i) $E_{\alpha}(x+y)=E_{\alpha}(x)+E_{\alpha}(y)$,
$x, y \in M_{+}$
(ii) $E_{\alpha}(\mu x)=\mu E_{\alpha}(x)$ $\mu \geq 0$
(iii) $x_{\iota} \uparrow x$ implies $E_{\alpha}\left(x_{\iota}\right) \uparrow E_{\alpha}(x)$
$x_{\iota} \in M_{+}$ 

(iv) $\alpha\left(\dot{E}_{\alpha}(z)\right)=\dot{E}_{\alpha}(z) \otimes 1_{G}$
$z \in \mathfrak{m}_{\alpha}$
(v) $\dot{E}_{\alpha}\left(b^{*} z b\right)=b^{*} \dot{E}_{\alpha}(z) b$
$b \in M_{\alpha}$.

Proof. (i), (ii) and (iii) are already shown in the above. We have only to prove (iv) and (v).

(iv) If $z \in \mathrm{m}_{\alpha}^{+}, \omega \in M_{*}^{+}$and $f \in L^{1}(G)_{+}$, then

$$
\begin{aligned}
& <\alpha\left(E_{\alpha}(z)\right), \omega \otimes f>=\sup _{g}\left\langle\alpha\left(\alpha_{g}(z)\right), \omega \otimes f>\right. \\
& \quad=\sup _{g}<\alpha(z), \omega \otimes(f * g)>\quad \text { By (2.5)) } \\
& =<\alpha(z), \omega \otimes \Delta><1_{G}, f>=<E_{\alpha}(z) \otimes 1_{G}, \omega \otimes f>.
\end{aligned}
$$

Since $\omega \otimes f$ are total in $\left(M \otimes L^{\infty}(G)\right)_{*}$, we have

$$
\alpha\left(\dot{E}_{\alpha}(x)\right)=\dot{E}_{\alpha}(x) \otimes 1_{G}
$$

for $x \in \mathrm{m}_{\alpha}$.

(v) If $b \in M_{\alpha}, x \in M_{+}$and $\omega \in M_{*}^{+}$, then

$$
\begin{aligned}
& <b E_{\alpha}(x) b^{*}, \omega>=<E_{\alpha}(x), b^{*} \omega b> \\
& =<\alpha(x),\left(b^{*} \omega b\right) \otimes \Delta>=<(b \otimes 1) \alpha(x)\left(b^{*} \otimes 1\right), \omega \otimes \Delta> \\
& =<\alpha\left(b x b^{*}\right), \omega \otimes \Delta>.
\end{aligned}
$$

Q.E.D.

Lemma 6.3. Let $\beta$ be $a$ dual action of $G$ on $N, M \equiv N \otimes{ }_{\beta}^{d} G$ and $\alpha \equiv \hat{\beta}$. Let $\mathrm{m}_{\alpha}$ and $M_{\alpha}$ be as in Lemma 6.2.

(i) If $g \in L^{1}(G) \cap L^{\infty}(G)$, then $1_{N} \otimes g \in \mathrm{m}_{\alpha}$ and $\dot{E}_{\alpha}\left(1_{N} \otimes g\right)=I(g) 1_{M}$.

(ii) $M_{\alpha} \mathfrak{m}_{\alpha} M_{\alpha} \subset \mathfrak{m}_{\alpha}$.

(iii) $\dot{E}_{\alpha}\left(\mathrm{m}_{\alpha}\right)=M_{\alpha}$.

Proof. (i) If $\xi \in \mathscr{K}$ and $f \in L^{2}(G)$, then

$$
\begin{aligned}
& <\alpha\left(1_{N} \otimes g\right), \omega_{\xi} \otimes \omega_{f} \otimes \Delta> \\
& =<1_{N} \otimes \varepsilon g, \omega_{\xi} \otimes|f|^{2} \otimes \Delta> \\
& \quad=<1_{N}, \omega_{\xi}>\iint g\left(t^{-1} s\right)|f(s)|^{2} \Delta(t) d s d t \\
& \quad=<1_{M}, \omega_{\xi} \otimes \omega_{f}>I(g) .
\end{aligned}
$$


Since $\omega_{\xi} \otimes \omega_{f}$ are total in $M_{*}, 1_{N} \otimes g \in \mathfrak{m}_{\alpha}$ and $\dot{E}_{\alpha}\left(1_{N} \otimes g\right)=I(g) 1_{M}$.

(ii) If $b \in M_{\alpha}, x \in \mathrm{n}_{\alpha}$ and $\omega \in M_{*}$, then

$$
\begin{aligned}
& <\alpha\left(b^{*} x^{*} x b\right), \omega \otimes \Delta> \\
& =<\left(b^{*} \otimes 1_{G}\right) \alpha\left(x^{*} x\right)\left(b \otimes 1_{G}\right), \omega \otimes \Delta> \\
& =<\alpha\left(x^{*} x\right), b \omega b^{*} \otimes \Delta> \\
& \leq \mu_{x}\left\|b \omega b^{*}\right\|=\mu_{x}\|\omega\|\|b\|
\end{aligned}
$$

by (6.6). Therefore $\mathfrak{n}_{\alpha} M_{\alpha} \subset \mathfrak{i}_{\alpha}$ and hence $M_{\alpha} \mathfrak{m}_{\alpha} M_{\alpha} \subset \mathfrak{m}_{\alpha}$.

(iii) Since $\dot{E}_{\alpha}\left(1 \mathrm{n}_{\alpha}\right) \subset M_{\alpha}$ by (iv) in Lemma 6.2, it suffices to show the converse inclusion. If $x \in M_{\alpha}$ and $g \in L^{1}(G) \cap L^{\infty}(G)$, then $\left(1_{N} \otimes g\right) x$ $\in \mathfrak{m}_{\alpha}$ by (ii) and

$$
\dot{E}_{\alpha}\left(\left(1_{N} \otimes g\right) x\right)=I(g) x
$$

by (v) of Lemma 6.2 and (i). Thus $x \in \dot{E}_{\alpha}\left(\mathrm{m}_{\alpha}\right)$.

Q.E.D.

Proposition 6.4. If $\beta$ is a dual action of $G$ on $N$, then $\beta(N)=$ $\left(N \otimes_{\beta}^{d} G\right)^{\bar{\beta}}$.

Proof. Let $M \equiv N \otimes_{\beta}^{d} G$ and $\alpha \equiv \hat{\beta}$. It is known that $M^{\alpha}=M_{\alpha}$. Indeed, $M^{\alpha} \subset M_{\alpha}$ is clear. If $x \in M_{\alpha}$, then $\alpha_{t}(x)=x$ locally almost everywhere in $t \in G$. Since $s \mapsto \alpha_{s}(x)$ is $\sigma$-strongly* continuous, $\alpha_{s}(x)=x$ for all s. Thus $x \in M^{\alpha}$.

Since $\beta(N) \subset M_{\alpha}$ by (2.12), it suffices to show the converse inclusion. We first notice that the mapping $y \in \mathrm{m}_{\alpha} \mapsto \dot{E}_{\alpha}\left(\left(1_{N} \otimes g\right) y\left(1_{N} \otimes g\right)\right) \in M_{\alpha}$ is $\sigma$ weakly continuous for each $g \in F$, where $F$ is a net in $K(G)_{+}$defining $I^{\prime}$ given at (6.3). This is because

$$
\left\|\alpha\left(1_{N} \otimes g\right)(\omega \otimes \Delta) \alpha\left(1_{N} \otimes g\right) \leq \mu_{1_{N} \otimes g}\right\| \omega \|
$$

by (6.6) and

$$
\begin{aligned}
& <\dot{E}_{\alpha}\left(\left(1_{N} \otimes g\right) y\left(1_{N} \otimes g\right)\right), \omega>=<\alpha\left(\left(1_{N} \otimes g\right) y\left(1_{N} \otimes g\right)\right), \omega \otimes \Delta> \\
& \quad=<\alpha(y), \alpha\left(1_{N} \otimes g\right)(\omega \otimes \Delta) \alpha\left(1_{N} \otimes g\right)>.
\end{aligned}
$$

Let $M_{0}$ be the linear span of 


$$
\int \beta(y(t))\left(1_{N} \otimes_{t-1} f\right) d t
$$

with $t \mapsto y(t)$ in $K(G, M)$ and $f \in K(G)$. The linear span $M_{1}$ of all $z^{*} y$ with $y, z \in M_{0}$ is $\sigma$-weakly dense in $M$. Since the convex cone $M_{1}^{+}$spanned by $y^{*} y$ with $y \in M_{0}$ generates linearly $M_{1}, M_{1}^{+}$is $\sigma$-weakly dense in $M_{+}$. Since $M_{1} \subset \mathrm{m}_{\alpha}$ by (i) and (ii) of Lemma 6.3, $M_{1}^{+}$is $\sigma$-weakly dense in $m_{\alpha}^{+}$. The $\sigma$-weak continuity shown in the above implies that $E_{\alpha}\left(\left(1_{N} \otimes g\right) M_{1}^{+}\left(1_{N} \otimes g\right)\right)$ is $\sigma$-weakly dense in $E_{\alpha}\left(\left(1_{N} \otimes g\right) \mathrm{m}_{\alpha}^{+}\left(1_{N} \otimes g\right)\right)$ for all $g \in F$. Since $E_{\alpha}$ is normal by (iii) in Lemma 6.2,

$$
\bigcup_{g \in F} E_{\alpha}\left(\left(1_{N} \otimes g\right) \mathrm{m}_{\alpha}^{+}\left(1_{N} \otimes g\right)\right)
$$

is $\sigma$-weakly dense in $E_{\alpha}\left(\mathrm{m}_{\alpha}^{+}\right)=M_{\alpha}^{+}$, which is due to (iii) of Lemma 6.3. Consequently, since $E_{\alpha}$ is normal,

$$
\bigcup_{g \in F} E_{\alpha}\left(\left(1_{N} \otimes g\right) M_{1}^{+}\left(1_{N} \otimes g\right)\right)
$$

is $\sigma$-weakly dense in $M_{\alpha}^{+}$. Since $E_{\alpha}\left(\left(1_{N} \otimes g\right) M_{1}^{+}\left(1_{N} \otimes g\right)\right)$ is included in $\beta(N)$ by Lemma 6.5 below, we have $M_{\alpha}^{+} \subset \beta(N)$, namely, $M_{\alpha} \subset \beta(N)$.

Q.E.D.

Lemma 6.5. $E_{\alpha}\left(\left(1_{N} \otimes g\right) \beta(x)\left(1_{N} \otimes g\right)\right) \in \beta(N)$.

Proof. Since $\left(1_{N} \otimes g\right) \beta(z)\left(1_{N} \otimes g\right) \in \mathrm{m}_{\alpha}$ for all $z \in N$, we may assume that $\operatorname{sp}_{\beta}(x)$ is compact by Lemma 4.5. Denote by $F$ the function on G:

$$
r \longmapsto \int g\left(t r^{-1}\right) g(t) \Delta(t) d t
$$

Then $F=\lambda_{*} \rho$ for some $\rho \in R(G)_{*}$. Therefore

$$
\begin{aligned}
& <E_{\alpha}\left(\left(1_{N} \otimes g\right) \beta(x)\left(1_{N} \otimes g\right)\right), \omega> \\
& \quad=<\alpha\left(\left(1_{N} \otimes g\right) \beta(x)\left(1_{N} \otimes g\right)\right), \omega \otimes \Delta> \\
& \quad=\lim <\int \beta_{\lambda(r)^{*} \phi}(x) \otimes<\lambda(r), \rho>\lambda(r) d r, \omega> \\
& \quad=<\beta\left(\beta_{\rho}(x)\right), \omega>.
\end{aligned}
$$

Q.E.D. 


\section{Duality for Crossed Dual Product by $\beta$}

We shall show another duality theorem for crossed product, which is also a generalization of Takesaki's duality.

Theorem 7.1. Let $N$ be a von Neumann algebra on $\mathscr{K}$. Let $\beta$ be a dual action of $G$ on $N, \alpha \equiv \hat{\beta}$ and $\tilde{\beta} \equiv \hat{\alpha}$. Let $\pi$ be a faithful representation of $N$ on $\mathscr{K} \otimes L^{2}(G) \otimes L^{2}(G)$ defined by

$$
\pi(x) \equiv\left(1 \otimes W^{\prime}\right)\left(\beta(x) \otimes 1_{G}\right)\left(1 \otimes W^{\prime}\right)^{*}
$$

for $x \in N$, where $W^{\prime}$ is defined by (1.9). Then

(i) $\left(N \otimes{ }_{\beta}^{d} G\right) \otimes_{\alpha} G$ is isomorphic to $N \otimes B\left(L^{2}(G)\right)$ and the isomorphism transforms $\pi(x)$ in the former to $x \otimes 1_{G}$ in the latter; and

(ii) $U^{*} \tilde{\beta}(\pi(x)) U=(\pi \otimes \iota) \beta(x)$,

where $U$ is defined on $\mathscr{K} \otimes L^{2}(G) \otimes L^{2}(G) \otimes L^{2}(G)$ by

$$
(U \xi)(r, s, t) \equiv \xi\left(r, s, t s^{-2} r^{2}\right) .
$$

Before going into the proof we shall prepare the following lemmas.

Lemma 7.2. (i) If $y_{r}$ is defined on $L^{2}(G) \otimes L^{2}(G)$ by $\quad \operatorname{Ad} W^{\prime}\left(1_{G} \otimes\right.$ $\left.\lambda^{\prime}(r)\right)$ or

$$
\left(y_{r} \xi\right)(s, t) \equiv \Delta(r)^{1 / 2} \xi\left(s, s r^{-1} s^{-1} t\right)
$$

then $y_{r}$ belongs to the von Neumann algebra generated by $\varepsilon L^{\infty}(G)$ and $1_{G} \otimes R(G)$, where $(\varepsilon f)(s, t) \equiv f\left(t^{-1} s\right)$.

(ii) $\operatorname{Ad} W^{\prime}\left(\lambda(r) \otimes \lambda^{\prime}(r)\right)=\lambda(r) \otimes 1_{G}$.

Proof. (i) For each $f$ and $g$ in $K(G)$ we set

$$
x_{f, g} \equiv \Delta(r)^{1 / 2} \int \varepsilon\left(a_{a^{-1}} g\right)\left(1_{G} \otimes \lambda\left(a r^{-1} a^{-1}\right)\right) f(a) d a .
$$

We may assume that $g \in K(G)$ and $\|\Delta g\|_{1}=1$. If $\xi \in K(G \times G)$, then

$$
\begin{aligned}
& \left(x_{f, g} \xi\right)(s, t) \\
& \left.\quad=\Delta(r)^{1 / 2} \int\left(\varepsilon_{a^{-1}} g\right)\left(1_{G} \otimes \lambda\left(a r^{-1} a^{-1}\right)\right) \xi\right)(s, t) f(a) d a
\end{aligned}
$$




$$
\begin{aligned}
& =\Delta(r)^{1 / 2} \int g\left(t^{-1} s a^{-1}\right) \xi\left(s, \operatorname{tar}^{-1} a^{-1}\right) f(a) d a \\
& =\int f\left(a t^{-1} s\right) g\left(a^{-1}\right) \Delta(r)^{1 / 2} \xi\left(s, t a t^{-1} s r^{-1} s^{-1} t a^{-1}\right) d a .
\end{aligned}
$$

Since $\left\|x_{f, g}\right\| \leq \Delta(r)^{1 / 2}\|f\|\|\Delta g\|_{1}$, when $(\Delta g)(a) d a$ converges to $\varepsilon_{e}, x_{f, g}$ converges weakly to $(\varepsilon f) y_{r}$. Since $x_{f, g}$ is in the von Neumann algebra $B_{0}$ generated by $\varepsilon L^{\infty}(G)$ and $1_{G} \otimes R(G)$, so is $(\varepsilon f) y_{r}$. Since $1_{G} \otimes 1_{G}$ is in the weak closure of $\varepsilon K(G), y_{r}$ belongs to $B_{0}$.

(ii) If $\xi \in L^{2}(G) \otimes L^{2}(G)$, then

$$
\begin{aligned}
& \left(W^{\prime}\left(\lambda(r) \otimes \lambda^{\prime}(r)\right) W^{\prime *} \xi\right)(s, t) \\
& \quad=\Delta(s r)^{1 / 2}\left(W^{\prime * \xi}\right)\left(s r, r^{-1} s^{-1} t\right) \\
& \quad=\xi(s r, t)=\left(\left(\lambda(r) \otimes 1_{G}\right) \xi\right)(s, t) .
\end{aligned}
$$

Q.E.D.

Lemma 7.3. Let $\phi$ and $\psi$ be elements in $R(G)_{*}$ with $\lambda_{*} \phi, \lambda_{*} \psi$ $\in K(G)$ and $\omega^{\prime}$ an element in $B\left(L^{2}(G) \otimes L^{2}(G)\right)_{*}$.

(i) The following four integrals exist as vector forms

$$
\begin{aligned}
& F_{\phi, \psi}^{1} \equiv \int\left(\lambda(r)^{*} \phi\right) \psi \otimes y_{r^{-1}} \omega^{\prime} d r \\
& F_{\phi, \psi}^{2} \equiv \int\left(\lambda(r)^{*} \phi\right) \psi \otimes \omega^{\prime}\left(1_{G} \otimes \lambda^{\prime}(r)\right) d r \\
& G_{\phi}^{1} \equiv \int<\lambda(r)^{*}, \phi>\left(\left(1_{G} \otimes \lambda^{\prime}(r)^{*}\right)\left(\omega^{\prime} \circ \mathrm{Ad} W^{\prime}\right)\right) d r \\
& G_{\phi}^{2} \equiv \int<\lambda(r)^{*}, \phi>\left(\left(\omega^{\prime} \circ \mathrm{Ad} W^{\prime *}\right) y_{r}\right) d r .
\end{aligned}
$$

(ii) If $\omega \in N_{*}$ and $y \in N$, then

$$
<\gamma \Phi_{\omega}(y) \otimes 1_{G}, F_{\phi, \psi}^{j}>=<\beta\left(\beta_{\psi}(y)\right) \otimes 1_{G}, \omega \otimes G_{\phi}^{j}>\quad(j=1,2) .
$$

Proof. The proof for $F_{\phi, \psi}^{2}$ and $G_{\phi}^{2}$ proceeds similarly as that for $F_{\phi, \psi}^{1}$ and $G_{\phi}^{1}$. We have only consider the latter.

(i) We first consider $F_{\phi, \psi}^{1}$. Since $r \mapsto \lambda(r)^{*} \phi$ and $r \mapsto y_{r-1} \omega^{\prime}$ are 
continuous by Lemma 4.2, $\quad r \mapsto\left(\lambda(r)^{*} \phi\right) \psi \otimes y_{r^{-1}} \omega^{\prime}$ is continuous. Since $\lambda_{*} \phi, \lambda_{*} \psi \in K(G)$ and $\operatorname{car} \lambda_{*}\left(\left(\lambda(r)^{*} \phi\right) \psi\right) \subset\left(\operatorname{car} \lambda_{*} \phi\right) r \cap \operatorname{car} \lambda_{*} \psi, r \mapsto\left(\lambda(r)^{*} \phi\right) \psi \otimes$ $y_{r^{-1}} \omega^{\prime}$ has a compact carrier. Therefore $F_{\phi, \psi}^{1}$ is Bochner integrable and hence it is the norm limit of vector forms.

As for $G_{\phi}^{1}$ we have only notice that $\lambda_{*} \phi \in K(G)$ and $r \mapsto\left(1_{G} \otimes \lambda^{\prime}(r)^{*}\right)$ $\left(\omega^{\prime} \circ \mathrm{Ad} W^{\prime}\right)$ is continuous.

(ii) We first show that

$$
<\gamma z \otimes 1_{G}, F_{\phi, \psi}^{1}>=<\gamma_{\psi} z \otimes 1_{G}, G_{\phi}^{1}>
$$

for all $z \in R(G)$. Since $F_{\phi, \psi}^{1}$ and $G_{\phi}^{1}$ are vector forms, it suffices to show (7.3) for all $\lambda(f)$ with $f \in L^{1}(G)$. Now, if $f \in L^{1}(G)$, then

$$
\begin{aligned}
& <\gamma \lambda(f) \otimes 1_{G}, F_{\phi, \psi}^{1}> \\
& =\int f(s)<\lambda(s) \otimes \lambda(s) \otimes 1_{G}, F_{\phi, \psi}^{1}>d s \\
& =\iint f(s)<\lambda(s), \psi><\lambda\left(s r^{-1}\right), \phi><\left(\lambda(s) \otimes 1_{G}\right) y_{r^{-1}}, \omega^{\prime}>d r d s \\
& =\iint f(s)<\lambda(s), \psi><\lambda(r)^{*}, \phi><\left(\lambda(s) \otimes 1_{G}\right) y_{s^{-1} r^{-1}}, \omega^{\prime}>d s d r \\
& =\iint f(s)<\lambda(s), \psi><\lambda(r)^{*}, \phi><\lambda(s) \otimes \lambda^{\prime}(r)^{*}, \omega^{\prime} \circ \mathrm{Ad} W^{\prime}>d s d r \\
& =\int<\lambda(r)^{*}, \phi><\gamma_{\psi} \lambda(f) \otimes \lambda^{\prime}(r)^{*}, \omega^{\prime} \circ \mathrm{Ad} W^{\prime}>d r \\
& =<\gamma_{\psi} \lambda(f) \otimes 1_{G}, G_{\phi}^{1}>,
\end{aligned}
$$

where the third equality is due to the Fubini theorem and the right invariance of Haar measure, and the fourth equality follows from Lemma 7.2 .

Now we replace $z$ in (7.3) by $\Phi_{\omega}(y)$. Then we have

$$
\begin{aligned}
& <\gamma \Phi_{\omega}(y) \otimes 1_{G}, F_{\phi, \psi}^{1}>=<\gamma_{\psi} \Phi_{\omega}(y) \otimes 1_{G}, G_{\phi}^{1}> \\
& =<\Phi_{\omega}\left(\beta_{\psi}(y)\right) \otimes 1_{G}, G_{\phi}^{1}> \\
& =<\beta\left(\beta_{\psi}(y)\right) \otimes 1_{G}, \omega \otimes G_{\phi}^{1}>,
\end{aligned}
$$


where the second equality is due to (ii) of Lemma 4.1. Thus (7.2) for $j=1$ is proved.

Q.E.D.

Proof of Theorem 7.1. Let $M \equiv N \otimes_{\beta}^{d} G$ and $D \equiv M \otimes_{\alpha} G$. Since $M$ is generated by $\beta(N)$ and $1 \otimes L^{\infty}(G)$ and since $D$ is generated by $\alpha(M)$ and $1_{M} \otimes R(G), D$ is generated by

$$
\beta(N) \otimes 1_{G}, \quad 1 \otimes \varepsilon L^{\infty}(G) \quad \text { and } \quad 1 \otimes 1_{G} \otimes R(G)
$$

by (2.12). Let $Q(f) \equiv 1 \otimes \varepsilon f$ and $\lambda_{2}(r) \equiv 1 \otimes 1_{G} \otimes \lambda(r)$ as in (1.10). Since

$$
\lambda_{2}(r) Q(f) \lambda_{2}(r)^{*}=Q\left(f_{r-1}\right)
$$

by direct culculation, $Q$ and $\lambda_{2}$ satisfy the commutation relation, [10]. Therefore the von Neumann algebra $B$ generated by $Q\left(L^{\infty}(G)\right)$ and $1 \otimes 1_{G} \otimes R(G)$ is isomorphic to $B\left(L^{2}(G)\right)$ and hence $D$ is isomorphic to $\left(D \cap B^{\prime}\right) \otimes B . \quad$ It is clear that $\lambda_{2}(r)$ commutes with $1 \otimes W^{\prime}$ and $\beta(x) \otimes 1_{G}$ and hence $\pi(x)$ commutes with $\lambda_{2}(r)$. Since for any $z \in N$ and $r \in G$

$$
\left(\left(1 \otimes W^{\prime}\right)\left(z \otimes \lambda(r) \otimes 1_{G}\right)\left(1 \otimes W^{\prime *}\right) \xi\right)(s, t)=\Delta(r)^{-1 / 2} z \xi\left(s r, s r s^{-1} t\right),
$$

it follows that $\operatorname{Ad} 1 \otimes W^{\prime}\left(z \otimes \lambda(r) \otimes 1_{G}\right)$ commutes with $Q(f)$ and hence that $\pi(x)$ commutes with $Q(f)$. Therefore $\pi(N) \subset B^{\prime}$.

Now, we shall show that $\pi(N) \subset D$. Choose $x \in N$ and $\phi, \psi \in R(G)_{*}$ with $\lambda_{*} \psi, \lambda_{*} \psi \in K(G)$. Since $r \mapsto \beta_{(\lambda(r) * \phi) \psi}(x)$ has a compact carrier, the integral

$$
\int\left(\beta\left(\beta_{\left(\lambda(r)^{*} \phi\right) \psi}(x)\right) \otimes 1_{G}\right)\left(1 \otimes y_{r-1}\right) d r
$$

exists for every $\phi \in R(G)_{*}$. We denote it by $x_{\phi, \psi}$. Then $x_{\phi, \psi}$ belongs to $D$ by (i) in Lemma 7.2. For any $\omega^{\prime}$ in $B\left(L^{2}(G) \otimes L^{2}(G)\right)_{*}$ we have

$$
\begin{aligned}
& <x_{\phi, \psi}, \omega \otimes \omega^{\prime}> \\
& =\int<\beta\left(\beta_{\left(\lambda(r)^{*} \phi\right) \psi}(x)\right) \otimes 1_{G}, \omega \otimes y_{r^{-1}} \omega^{\prime}>d r \\
& =<\gamma \Phi_{\omega}(x) \otimes 1_{G}, F_{\phi, \psi}^{1}> \\
& =<\beta\left(\beta_{\psi}(x)\right) \otimes 1_{G}, \omega \otimes G_{\phi}^{1}> \\
& =<\operatorname{Ad} 1 \otimes W^{\prime}\left(\beta\left(\beta_{\psi}(x)\right) \otimes \int<\lambda(r)^{*}, \phi>\lambda^{\prime}(r)^{*} d r\right), \omega \otimes \omega^{\prime}>
\end{aligned}
$$


where the second equality follows from (iii) of Lemma 4.1 and the third equality follows from Lemma 7.3. Since we may assume that $\lambda_{*} \phi \in K(G)_{+}$and $\left\|\Delta \lambda_{*} \phi\right\|_{1}=1$, the norm of the first argument of the right hand side of (7.5) is majorized by $\left\|\beta_{\psi}(x)\right\|$. Further, since $\omega \otimes \omega^{\prime}$ are total in the set of all vector forms, $x_{\phi, \psi}$ is bounded by $\left\|\beta_{\psi}(x)\right\|$. Since $\omega \otimes \omega^{\prime}$ are total in $D_{*},(7.5)$ shows that $x_{\phi, \psi}$ converges $\sigma$-weakly to

$$
\operatorname{Ad} 1 \otimes W^{\prime}\left(\beta\left(\beta_{\psi}(x)\right) \otimes 1_{G}\right)=\pi\left(\beta_{\psi}(x)\right)
$$

as $\left\langle\lambda(r)^{*}, \phi\right\rangle d r$ tends to $\varepsilon_{e}$. Since $x_{\phi, \psi} \in D, \pi\left(\beta_{\psi}(x)\right) \in D$. Since $x$ is in the von Neumann algebra generated by $\beta_{\psi}(x)$ with $\psi \in R(G)_{*}$ and $\lambda_{*} \psi \in K(G)$ by Lemma $4.5, \pi(x)$ belongs to $D$.

Next, we shall show that $\beta(N) \otimes I_{G}$ is included in $(\pi(N) \cup B)^{\prime \prime}$. For cach $y \in N$ we denote by $y_{\phi, \psi}$ an element of the form

$$
\int \beta\left(\beta_{\left(\lambda(r)^{*} \phi\right) \psi}(y)\right) \otimes \lambda^{\prime}(r) d r
$$

where $\phi, \psi$ are in $R(G)_{*}$ with $\lambda_{*} \phi, \lambda_{*} \psi \in K(G)$. Since $y_{r}=\operatorname{Ad} W^{\prime}\left(1_{G} \otimes\right.$ $\lambda^{\prime}(r)$ ) by (i) in Lemma 7.2, $\operatorname{Ad} 1 \otimes W^{\prime}\left(y_{\phi, \psi}\right)$ belongs to $(\pi(N) \cup B)^{\prime \prime}$. For any $\omega^{\prime}$ in $B\left(L^{2}(G) \otimes L^{2}(G)\right)_{*}$ we have

$$
\begin{aligned}
< & y_{\phi, \psi},(1) \otimes \omega^{\prime}> \\
& =\int<\beta\left(\beta_{\left(\lambda(r)^{*} \phi\right) \psi}(y)\right) \otimes 1_{G}, \omega \otimes \omega^{\prime}\left(1_{G} \otimes \lambda^{\prime}(r)\right)>d r \\
(7.6) \quad & =<\gamma \Phi_{\omega}(y) \otimes 1_{G}, F_{\phi, \psi}^{2}> \\
& =<\beta\left(\beta_{\psi}(y)\right) \otimes 1_{G}, \omega \otimes G_{\phi}^{2}> \\
& =<\operatorname{Ad} 1 \otimes W^{\prime *}\left(\beta\left(\beta_{\psi}(y)\right) \otimes 1_{G} \int<\lambda(r)^{*}, \phi>1 \otimes y_{r} d r\right), \omega \otimes \omega^{\prime}>,
\end{aligned}
$$

where the second and third equalities follow from Lemmas 4.1 and 7.3, respectively. Since we may assume that $\lambda_{*} \phi \in K(G)_{+}$and $\left\|\Delta \lambda_{*} \phi\right\|_{1}=1$, $y_{\phi, \psi}$ is bounded by $\left\|\beta_{\psi}(y)\right\|$ by a similar reason as before. Since $\omega \otimes \omega^{\prime}$ are total in the predual of $\operatorname{Ad} 1 \otimes W^{* *}(\pi(N) \cup B)^{\prime \prime}$, (7.6) shows that $y_{\phi, \psi}$ converges $\sigma$-weakly to 
$\operatorname{Ad} 1 \otimes W^{\prime *}\left(\beta\left(\beta_{\psi}(y)\right) \otimes 1_{G}\right)$,

as $\left\langle\lambda(r)^{*}, \phi>d r\right.$ tends to $\varepsilon_{e}$. Since $\operatorname{Ad} 1 \otimes W^{\prime}\left(y_{\phi, \psi}\right)$ belongs to $(\pi(N)$ $\cup B)^{\prime \prime}, \beta\left(\beta_{\psi}(y)\right) \otimes 1_{G} \quad$ belongs to $(\pi(N) \cup B)^{\prime \prime}$ and hence $\beta(y) \otimes 1_{G} \in(\pi(N)$ $\cup B)^{\prime \prime}$.

Consequently, we have shown both that $\pi(N) \cup B \subset D$ and that $\left(\beta(N) \otimes 1_{G}\right) \cup B \subset(\pi(N) \cup B)^{\prime \prime}$. Since $D$ is generated by $\beta(N) \otimes 1_{G}$ and $B$, we have $D=(\pi(N) \cup B)^{\prime \prime}$. Since $\pi(N) \subset D \cap B^{\prime}, D$ is isomorphic to $\pi(N)$ $\otimes B$.

(ii) Put $U_{0} \equiv U^{*}\left(1 \otimes 1_{G} \otimes W\right)^{*}\left(1 \otimes W^{\prime} \otimes 1_{G}\right)$. Then

$$
\left(U_{0} \xi\right)(r, s, t)=\Delta(r)^{1 / 2} \xi\left(r, r^{-1} s, t r^{-2} s\right) .
$$

Here, by Lemma 4.5, we have only to show (ii) for $x$ with compact $\operatorname{sp}_{\beta}(x)$. If $\phi \in R(G)_{*}$ and $\lambda_{*} \phi \in K(G)$, then for any $a \in G$

$$
\begin{aligned}
& \left(\left(\int \beta_{\lambda(a)^{*} \phi}(x) \otimes \lambda(a) \otimes 1_{G} \otimes 1_{G} d a\right) U_{0}^{*} \xi\right)\left(r, r^{-1} s, t r^{-2} s\right) \\
& \quad=\int \beta_{\lambda(a)^{*} \phi}(x)\left(U_{0}^{*} \xi\right)\left(r a, r^{-1} s, t r^{-2} s\right) d a \\
& =\int \beta_{\lambda(a)^{*} \phi}(x) \Delta(r a)^{-1 / 2} \xi\left(r a, r a r^{-1} s, t a\right) d a \\
& =\int \beta_{\lambda(a)^{*} \phi}(x)\left(\left(1 \otimes W^{\prime} \otimes 1_{G}\right)^{*} \zeta\right)\left(r a, r^{-1} s, t a\right) d a \\
& =\left(\left(\beta_{\lambda(a)^{*} \phi}(x) \otimes \lambda(a) \otimes 1_{G} \otimes \lambda(a) d a\right)\left(1 \otimes W^{\prime} \otimes 1_{G}\right)^{* \xi}\right)\left(r, r^{-1} s, t\right)
\end{aligned}
$$

Here we assume that $\omega_{\xi}$ belongs to the algebraic tensor product $N_{*} \odot$ $R(G)_{*} \odot R(G)_{*} \odot R(G)_{*}$. By virtue of (ii) in Lemma 4.3 if $\left\langle\lambda(a)^{*}, \phi\right\rangle d a$ converges to $\varepsilon_{e}$, the left hand side of (7.8) multiplied by $\Delta(r)^{1 / 2}$ converges to

$$
\begin{aligned}
& \Delta(r)^{1 / 2}\left(\left(\beta(x) \otimes 1_{G} \otimes 1_{G}\right) U_{0}^{*} \xi\right)\left(r, r^{-1} s, t r^{-2} s\right) \\
& \quad=\left(U_{0}\left(\beta(x) \otimes 1_{G} \otimes 1_{G}\right) U_{0}^{*} \xi\right)(r, s, t) \\
& =\left(U^{*} \tilde{\beta}(\pi(x)) U \xi\right)(r, s, t) .
\end{aligned}
$$

Define a unitary $U_{1}$ on $\mathscr{K} \otimes L^{2}(G) \otimes L^{2}(G) \otimes L^{2}(G)$ by 


$$
\left(U_{1} \xi\right)(r, s, t) \equiv \xi(r, t, s)
$$

and put $U_{2} \equiv\left(1 \otimes W^{\prime} \otimes 1_{G}\right) U_{1}$. Then the right hand side of (7.8) multiplied by $\Delta(r)^{1 / 2}$ converges to

$$
\begin{aligned}
& \Delta(r)^{1 / 2}\left(U_{1}\left((\iota \otimes \gamma) \beta(x) \otimes 1_{G}\right) U_{1}^{*}\left(1 \otimes W^{\prime} \otimes 1_{G}\right)^{*} \xi\right)\left(r, r^{-1} s, t\right) \\
& =\left(U_{2}\left((\iota \otimes \gamma) \beta(x) \otimes 1_{G}\right) U_{2}^{*} \xi\right)(r, s, t) \\
& =\left(U_{2}\left((\beta \otimes \iota) \beta(x) \otimes 1_{G}\right) U_{2}^{*} \xi\right)(r, s, t) \\
& =(((\pi \otimes \iota) \beta(x)) \xi)(r, s, t) .
\end{aligned}
$$

Since the set of $\zeta$ considered there is dense in $\mathscr{K} \otimes L^{2}(G) \otimes L^{2}(G) \otimes L^{2}(G)$, we complete the proof of (ii).

Combining Proposition 6.4 and Theorem 7.1, we have the following corollary.

Corollary 7.4. If $M$ is of the form $N \otimes_{\beta}^{d} G$ for some $N$ and a dual action $\beta$ of $G$ on $N$, then

(i) $M^{\alpha}=\beta(N)$ for $\alpha \equiv \hat{\beta}$; and

(ii) $M \otimes_{\alpha} G$ is isomorphic to $M^{\alpha} \otimes B\left(L^{2}(G)\right)$.

The existence of a pair $\{N, \beta\}$ in the above corollary is always assured by Theorem 3.1 whenever $M$ is properly infinite and $G$ is separable.

Combining Theorem 3.1 and Proposition 6.1, we have the following corollary.

Corollary 7.5. If $N$ is of the form $M \otimes_{\alpha} G$ for some $M$ and an action $\alpha$ of $G$ on $M$, then

(i) $N^{\beta}=\alpha(M)$ for $\beta \equiv \hat{\alpha}$; and

(ii) $N \otimes{ }_{\beta}^{d} G$ is isomorplic to $N^{\beta} \otimes B\left(L^{2}(G)\right)$.

The existence of a pair of $\{M, \alpha\}$ in Corollary 7.5 is assured by Theorem 7.1 whenever $N$ is properly infinite and $G$ is separable. 


\section{Haga's Factorization of Crossed Product}

In this section we shall establish a structure theorem of a crossed dual product corresponding to the Landstad's theorem which gives a necessary and sufficient condition for a given von Neumann algebra to be a crossed product with respect to a given locally compact group. Combining this theorem with the Takesaki's duality of second type, we can give a sufficient condition under which a Haga's factorization for a crossed product is possible.

Theorem 8.1 (Landstad [9]). Let $N$ be a von Neumann algebra and $G$ a locally compact group. The following two conditions are equivalent:

(i) there exist a von Neumann algebra $M$ and an action $\alpha$ of $G$ on $M$ satisfying $N \sim M \otimes_{\alpha} G$; and

(ii) there exist a weakly continuous unitary representation $u$ of $G$ in $N$ and a dual action $\beta$ of $G$ on $N$ satisfying $\beta(u(t))=u(t) \otimes$ $\lambda(t)$ for all $t \in G$.

Proof. (i) $\Rightarrow$ (ii) We may assume that $N=M \otimes_{\alpha} G$. If we put $\beta \equiv \hat{\alpha}$ and $u(t) \equiv \lambda_{1}(t)$, then (ii) follows from (2.9).

(ii) $\Rightarrow$ (i) Let $\mathrm{nt}_{\beta}$ and $N_{\beta}$ be as in Section 6 . Let $F$ be an increasing net given at (6.2), namely, $\omega_{e}=\sup \{\omega: \omega \in F\}$.

First we shall show that $N$ is generated by $N_{\beta}$ and $u(t), t \in G$. If $\phi \in F, y \in \mathfrak{m}_{\beta}^{+}, \omega \in N_{*}^{+}$and $\psi \in R(G)_{*}^{+}$, then

$$
\begin{aligned}
< & \beta\left(\beta_{\phi}\left(y u(t)^{*}\right) u(t)\right), \omega \otimes \psi> \\
& =<\beta\left(\beta_{\phi}\left(y u(t)^{*}\right)\right), u(t) \omega \otimes \lambda(t) \psi> \\
& =<\beta\left(y u(t)^{*}\right), u(t) \omega \otimes \phi(\lambda(t) \psi)> \\
& =<\beta(y), \omega \otimes\left(\lambda(t)^{*} \phi\right) \psi>
\end{aligned}
$$

Since we may assume that $\left\langle\lambda(t)^{*}, \phi\right\rangle d t$ converges to $\varepsilon_{e}$,

$$
<\int \beta\left(\dot{E}_{\beta}\left(y u(t)^{*}\right) u(t)\right) d t, \omega \otimes \psi>=<\beta(y), \omega \otimes \psi>
$$

by (8.1) and hence 


$$
y=\int \dot{E}_{\beta}\left(y u(t)^{*}\right) u(t) d t
$$

Since $\mathfrak{m}_{\beta}^{+}$is $\sigma$-weakly dense in $N_{+}, N$ is generated by $N_{\beta}$ and $u(t)$, $t \in G$ by the fact that $\dot{E}_{\beta}\left(\mathrm{m}_{\beta}\right)=N_{\beta}$.

Next, we notice that $\operatorname{Ad} u(t)$ is an action of $G$ on $N_{\beta}$. Indeed, if $x \in N_{\beta}$, then

$$
\beta\left(u(t) x u(t)^{*}\right)=\operatorname{Ad} u(t) \otimes \lambda(t)\left(x \otimes 1_{G}\right)=u(t) x u(t)^{*} \otimes 1_{G}
$$

and hence $\operatorname{Ad} u(t)\left(N_{\beta}\right)=N_{\beta}$.

Finally we shall show that $N$ is isomorphic to $M \otimes_{\alpha} G$, where $M$ $\equiv N_{\beta}$ and $\alpha(x) \equiv \operatorname{Ad} u\left(x \otimes 1_{G}\right)$ for $x \in M$. Since

$$
\begin{aligned}
& \left(u^{*}\left(1_{M} \otimes \lambda(t)\right) u \xi\right)(s)=u(s)^{*}(u \xi)(s t) \\
& \quad=u(t) \xi(s t)=((u(t) \otimes \lambda(t)) \xi)(s)=(\beta(u(t)) \xi)(s)
\end{aligned}
$$

for $\xi$ in $L^{2}(G, \mathscr{K})$ and

$$
u^{*} \alpha(x) u=x \otimes 1_{G}=\beta(x)
$$

for $x$ in $M \equiv N_{\beta}$, we have $M \otimes_{\alpha} G=\operatorname{Ad} u \circ \beta(N)$ and hence $N$ is isomorphic to $M \otimes_{\alpha} G$.

Q.E.D.

If we combine Corollary 7.5 and Theorem 8.1 , we will have the following theorem.

Theorem 8.2. Let $\beta$ be a dual action of $G$ on $N$. If there exists a weakly continuous unitary representation $u$ of $G$ in $N$ satisfying $\beta(u(t))=u(t) \otimes \lambda(t)$ for all $t \in G$, then $N \otimes_{\beta}^{d} G$ is isomorphic to $N^{\beta} \otimes$ $B\left(L^{2}(G)\right)$.

Proof. According to Theorem 8.1 there exists a von Neumann algebra $M$ and an action $\alpha$ of $G$ on $M$ such that $N$ is isomorphic to $M \otimes_{\alpha} G$. Put $\rho \equiv \operatorname{Ad} u \circ \beta$. Using (8.2) and (8.3), we have $\rho(u(t))=$ $\lambda_{1}(t)$ and $\rho(x)=\alpha(x)$ for $x \in N_{\beta}$. Since

$$
\begin{gathered}
(\rho \otimes \imath) \beta(x)=\rho(x) \otimes 1_{G}=\alpha(x) \otimes 1_{G} \\
=\hat{\alpha}(\alpha(x))=\hat{\alpha}(\rho(x))
\end{gathered}
$$

for $x \in N_{\beta}$ and 


$$
\begin{aligned}
& (\rho \otimes \imath) \beta(u(t))=(\rho \otimes \iota)(u(t) \otimes \lambda(t))=\lambda_{1}(t) \otimes \lambda(t) \\
& =\hat{\alpha}\left(\lambda_{1}(t)\right)=\hat{\alpha}(\rho(u(t))),
\end{aligned}
$$

we know that $\left(\rho \otimes_{\ell}\right) \circ=\hat{\alpha} \circ \rho$ and hence that $\beta$ is a dual action dual to $\alpha$ through the isomorphism $\rho$. Therefore by Corollary 7.5 we complete the proof.

Theorem 8.3. Let $M$ be a von Neumann algebra and $G$ a locally compact group. The following three conditions are equivalent:

(i) there exist a von Neumann algebra $N$ and a dual action $\beta$ of $G$ on $N$ satisfying $M \sim N \otimes{ }_{\beta}^{d} G$;

(ii) there exists an action $\alpha$ of $G$ on $M$ and a Hilbert space $\mathscr{K}$ such that $1_{\mathscr{K}} \otimes L^{\infty}(G) \subset M$ and $\alpha\left(1_{\mathscr{K}} \otimes f\right)=1_{\mathscr{H}} \otimes \varepsilon f\left(\right.$ or $\quad \alpha_{t}\left(1_{\mathscr{K}} \otimes f\right)=1_{\mathscr{K}} \otimes$ $f_{t^{-1}}$ for all $\left.t \in G\right)$; and

(iii) (assume that $M$ is standard) there exist an action a of $G$ on $M$ and a weakly continuous unitary representation $v$ on a Hilbert space $\mathscr{K}$ such that $1_{\mathscr{K}} \otimes L^{\infty}(G) \subset M$ and $\alpha_{t}=\operatorname{Ad} v(t) \otimes \lambda^{\prime}(t) \uparrow M$ for all $t \in G$.

Proof. (i) $\Rightarrow$ (ii) We may assume that $M \equiv N \otimes_{\beta}^{d} G$. Put $\alpha \equiv \hat{\beta}$. Then $1_{N} \otimes L^{\infty}(G) \subset M$ and $\alpha\left(1_{N} \otimes f\right)=1_{N} \otimes \varepsilon f$ by (2.12). Therefore

$$
\begin{aligned}
& \alpha_{t}\left(1_{N} \otimes f\right) \dot{\zeta}(t)=\left(\alpha\left(1_{N} \otimes f\right) \xi\right)(t) \\
& \quad=\left(\left(1_{N} \otimes \varepsilon f\right) \xi\right)(t)=\left(1_{N} \otimes f_{t^{-1}}\right) \check{\zeta}(t)
\end{aligned}
$$

and so $\alpha_{t}\left(1_{N} \otimes f\right)=1_{N} \otimes f_{t^{-1}}$ for all $t \in G$.

(ii) $\Rightarrow$ (iii) We have only to show the existence of a weakly continuous unitary representation $v$ on $\mathscr{K}$ such that $\alpha_{t}=\operatorname{Ad} v(t) \otimes \lambda^{\prime}(t)$ on $M$.

Now we may assume that $\alpha_{t}$ is implemented by a weakly continuous unitary representation $u$ of $G$ on $\mathscr{K} \otimes L^{2}(G)$ by considering $M$ to be standard. Since by (ii)

$$
\begin{aligned}
& \operatorname{Ad} u(r)\left(1_{\mathscr{K}} \otimes f\right)=\alpha_{r}\left(1_{\mathscr{K}} \otimes f\right) \\
&=1_{\mathscr{K}} \otimes f_{r^{-1}}=\operatorname{Ad} 1_{\mathscr{K}} \otimes \lambda^{\prime}(r)\left(1_{\mathscr{K}} \otimes f\right)
\end{aligned}
$$

for all $f \in L^{\infty}(G)$, we have $\left(1_{\mathscr{K}} \otimes \lambda^{\prime}(r)\right)^{*} u(r) \in B(\mathscr{K}) \otimes L^{\infty}(G)$. Therefore there is an essentially bounded weakly mcasurable function $r \in G \mapsto v(r)$ in $L^{\infty}(G, B(\mathscr{K}))$ such that $v(r)$ are unitaries on $\mathscr{K}$ for all $r \in G$ and $u(r)$ 
$=v(r) \otimes \lambda^{\prime}(r)$. Since $u$ is a representation of $G$, so is $v$. The continuity of unitary representation is immediate from measurability.

(iii) $\Rightarrow$ (i) Let $n_{\alpha}$ and $N_{\beta}$ be as in Section 6 . Let $F$ be an increasing net given at (6.3), namely $I^{\prime}=\sup \{g: g \in F\}$.

First we shall show that $M$ is generated by $M_{\alpha}$ and $1_{\mathscr{H}} \otimes L^{\infty}(G)$. Suppose that $y \in \mathrm{m}_{\alpha}$. If $k \in K(G), g \in F, \omega \in B(\mathscr{K})_{*}, h \in L^{2}(G)$ and $f \in$ $L^{1}(G)$, then

$$
\begin{aligned}
& <\alpha\left(\left(1_{\mathscr{H}} \otimes_{r^{-1}} k\right) \alpha_{g}\left(\left(1_{\mathscr{H}} \otimes_{r^{-1}} k\right) y\right)\right), \omega \otimes \omega_{h} \otimes f> \\
& =<\alpha\left(\alpha_{g}\left(\left(1_{\mathscr{H}} \otimes_{r^{-1}} k\right) y\right)\right), \omega \otimes \omega_{h \otimes 1_{G},\left(\varepsilon_{r}-1 k\right)(h \otimes f)}> \\
& =<\alpha\left(\left(1_{\mathscr{H}} \otimes_{r^{-1}} k\right) y\right), \omega \otimes \omega_{h \otimes 1_{G},\left(\left(\varepsilon_{r}-1 k\right)(h \otimes f)\right) \hat{*}\left(1_{G} \otimes g\right)}> \\
& =<\alpha(y), \omega \otimes \omega_{h \otimes 1_{G}, l_{r}}>
\end{aligned}
$$

where $\hat{*}$ indicates the convolution product with respect to the second argument, $\omega_{h \otimes 1_{G}, K}(\lambda(a) \otimes H) \equiv I \otimes I\left(\bar{K}\left({ }_{a} h \otimes H\right)\right)$ for $H \in L^{\infty}(G)$ and

$$
l_{r} \equiv\left(\varepsilon_{r^{-1}} k\right)\left\{\left(\left(\varepsilon_{r^{-1}} k\right)(h \otimes f)\right) \hat{*}\left(1_{G} \otimes g\right)\right\} .
$$

Since

$$
\begin{aligned}
l_{r}(s, t) & =k\left(t^{-1} s r^{-1}\right) \int\left(\left(\varepsilon_{r^{-1}} k\right)(h \otimes f)\right)\left(s, t b^{-1}\right) g(b) d b \\
& =k\left(t^{-1} s r^{-1}\right) \int k\left(b t^{-1} s r^{-1}\right) h(s) f\left(t b^{-1}\right) g(b) d b \\
& =k\left(t^{-1} s r^{-1}\right) \int k(b) h(s) f\left(s r^{-1} b^{-1}\right) g\left(b r s^{-1} t\right) d b
\end{aligned}
$$

we have, by right invariance of Haar measure,

$$
\begin{aligned}
\int l_{r}(s, t) d r & =\int k\left(r^{-1}\right) \int k(b) h(s) f\left(t r^{-1} b^{-1}\right) g(b r) d b d r \\
& =\left(h \otimes \iint k\left(r^{-1}\right) k\left(b r^{-1}\right) g(b)_{b^{-1}} f d r d b\right)(s, t) \\
& =(h \otimes(f *((k * \check{k}) g)))(s, t),
\end{aligned}
$$

where $\check{k}(r) \equiv k\left(r^{-1}\right)$. Therefore 


$$
\begin{aligned}
& \int<\alpha\left(\left(1_{\mathscr{r}} \otimes_{r^{-1}} k\right) \alpha_{g}\left(\left(1_{\mathscr{N}} \otimes_{r^{-1}} k\right) y\right)\right), \omega \otimes \omega_{h} \otimes f>d r \\
& =<\alpha(y), \omega \otimes \omega_{h} \otimes(f *((k * \check{k}) g))>
\end{aligned}
$$

by (8.4). If $g \in F$ converges to $\Delta$ in the compact convergence topology,

$$
\begin{aligned}
& <\alpha\left(\int\left(1_{\mathscr{X}} \otimes_{r^{-1}} k\right) E_{\alpha}\left(\left(1_{\mathscr{K}} \otimes_{r^{-1}} k\right) y\right) d r\right), \omega \otimes \omega_{h} \otimes f> \\
& \quad=<\alpha(y), \omega \otimes \omega_{h} \otimes(f *((k \otimes \check{k}) \Delta))>
\end{aligned}
$$

by (8.5). If $(k * \check{k})(r) d r$ converges to $\varepsilon_{e}$, then

$$
\int\left(1_{\mathscr{K}} \otimes_{r^{-1}} k\right) E_{\alpha}\left(\left(1_{\mathscr{H}} \otimes_{r^{-1}} k\right) y\right) d r
$$

converges $\sigma$-weakly to $y$. Since the element at (8.6) belongs to the von Neumann algebra generated by $M_{\alpha}$ and $1_{\mathscr{K}} \otimes L^{\infty}(G)$, so does $y$. Since $\mathfrak{m}_{\alpha}$ is $\sigma$-weakly dense in $M$ as in the proof of Proposition 6.4, $M$ is generated by $M_{\alpha}$ and $1_{\mathscr{K}} \otimes L^{\infty}(G)$.

Now we define a unitary $w$ on $\mathscr{K} \otimes L^{2}(G) \otimes L^{2}(G)$ by

$$
(w \xi)(s, t) \equiv \Delta\left(t s^{-1}\right)^{1 / 2} \xi\left(s, s t^{-1}\right)
$$

and an isomorphism $\beta$ by

$$
\beta(x) \equiv \operatorname{Ad} w\left(x \otimes 1_{G}\right)
$$

for all $x$ in $M_{\alpha^{*}}$ Then $\left(w^{*} \xi\right)(s, t)=\Delta(t)^{1 / 2} \xi\left(s, t^{-1} s\right)$. We shall show that $\beta$ is an isomorphism of $M_{\alpha}$ into $M_{\alpha} \otimes R(G)$.

Since $w^{*}\left(1_{M} \otimes \lambda^{\prime}(r)\right) w=1_{M} \otimes \lambda(r)$ by

$$
\begin{aligned}
& \left(w^{*}\left(1_{M} \otimes \lambda^{\prime}(r)\right) w \xi\right)(s, t)=\Delta(t)^{1 / 2}\left(\left(1_{M} \otimes \lambda^{\prime}(r)\right) w \xi\right)\left(s, t^{-1} s\right) \\
& \quad=\Delta(t r)^{1 / 2}(w \xi)\left(s, r^{-1} t^{-1} s\right)=\xi(s, t r)=\left(\left(1_{M} \otimes \lambda(r)\right) \xi\right)(s, t),
\end{aligned}
$$

we have, for any $x$ in $M_{\alpha}$,

$$
\begin{aligned}
& w\left(x \otimes 1_{G}\right) w^{*}\left(1_{M} \otimes \lambda^{\prime}(r)\right)=w\left(x \otimes 1_{G}\right)\left(1_{M} \otimes \lambda(r)\right) w^{*} \\
& \quad=w\left(1_{M} \otimes \lambda(r)\right)\left(x \otimes 1_{G}\right) w^{*}=\left(1_{M} \otimes \lambda^{\prime}(r)\right) w\left(x \otimes 1_{G}\right) w^{*}
\end{aligned}
$$

and hence 


$$
w\left(x \otimes 1_{G}\right) w^{*} \in B\left(\mathscr{K} \otimes L^{2}(G)\right) \otimes R(G) .
$$

Since $M^{\prime} \subset B(\mathscr{K}) \otimes L^{\infty}(G)$ by assumption (iii), if $y \in M^{\prime}$, then $y$ is an essentially bounded weakly measurable function $r \mapsto y^{\prime}(r)$ in $L^{\infty}(G, B(\mathscr{K}))$ and hence $\left[w, y \otimes 1_{G}\right]=0$, for

$$
\begin{aligned}
& \left(w\left(y \otimes 1_{G}\right) \xi\right)(s, t)=\Delta\left(t s^{-1}\right)^{1 / 2}\left(\left(y \otimes 1_{G}\right) \xi\right)\left(s, s t^{-1}\right) \\
& \quad=\Delta\left(t s^{-1}\right)^{1 / 2} y(s) \xi\left(s, s t^{-1}\right)=y(s)(w \xi)(s, t) \\
& \quad=\left(\left(y \otimes 1_{G}\right) w \xi\right)(s, t) .
\end{aligned}
$$

Therefore, for any $x$ in $M_{\alpha}$,

and hence by (8.7)

$$
\left[w\left(x \otimes 1_{G}\right) w^{*}, y \otimes 1_{G}\right]=0
$$

$$
w\left(x \otimes 1_{G}\right) w^{*} \in M \otimes R(G) .
$$

Here we set $u(t) \equiv v(t) \otimes \lambda^{\prime}(t)$ on $\mathscr{K} \otimes L^{2}(G)$. Since $w^{*}\left(u(r) \otimes 1_{G}\right) w=u(r)$ $\otimes \lambda^{\prime}(r)$ by

$$
\begin{aligned}
& \left(w^{*}\left(u(r) \otimes 1_{G}\right) w \xi\right)(s, t)=\Delta(t)^{1 / 2}\left(\left(u(r) \otimes 1_{G}\right) w \xi\right)\left(s, t^{-1} s\right) \\
& \quad=\Delta(t r)^{1 / 2} v(r)(w \xi)\left(r^{-1} s, t^{-1} s\right) \\
& \quad=\Delta(r) v(r) \xi\left(r^{-1} s, r^{-1} t\right)=\left(\left(u(r) \otimes \lambda^{\prime}(r)\right) \xi\right)(s, t),
\end{aligned}
$$

we have, for any $x$ in $M_{\alpha}$,

$$
\begin{aligned}
& \left(u(r) \otimes 1_{G}\right)\left(w\left(x \otimes 1_{G}\right) w^{*}\right)\left(u(r) \otimes 1_{G}\right)^{*} \\
& =w\left(u(r) \otimes \lambda^{\prime}(r)\right)\left(x \otimes 1_{G}\right)\left(u(r) \otimes \lambda^{\prime}(r)\right)^{*} w^{*} \\
& =w\left(\alpha_{r}(x) \otimes 1_{G}\right) w^{*}=w\left(x \otimes 1_{G}\right) w^{*} .
\end{aligned}
$$

Combining (8.8) and (8.9), we have

$$
w\left(x \otimes 1_{G}\right) w^{*} \in M_{\alpha} \otimes R(G),
$$

which shows that $\beta$ is an isomorphism of $M_{\alpha}$ into $M_{\alpha} \otimes R(G)$.

Next we shall show that $\beta$ is a dual action of $G$ on $M_{\alpha}$. Define a unitary $U_{1}$ on $\mathscr{K} \otimes L^{2}(G) \otimes L^{2}(G) \otimes L^{2}(G)$ by 


$$
\left(U_{1} \xi\right)(r, s, t) \equiv \xi(r, t, s)
$$

as (7.9) and put $\check{w} \equiv U_{1}\left(w \otimes 1_{G}\right) U_{1}^{*}$. For $z$ in $K(G, B(\mathscr{K}))$ we put

$$
\tilde{z} \equiv \int z(a) \otimes \lambda(a) d a
$$

Since

$$
\begin{aligned}
& \operatorname{Ad}\left(\left(w \otimes 1_{G}\right) \check{w}\right)\left(\tilde{z} \otimes 1_{G} \otimes 1_{G}\right) \\
& =\int z(a) \otimes \lambda(a) \otimes \lambda(a) \otimes \lambda(a) d a
\end{aligned}
$$

and

$$
\operatorname{Ad} w\left(\tilde{z} \otimes 1_{G}\right)=\int z(a) \otimes \lambda(a) \otimes \lambda(a) d a
$$

by direct culculation, we have

$$
\operatorname{Ad}(w \otimes 1) \check{w} \circ \iota \otimes \gamma=\iota \otimes \gamma \circ A d w
$$

on $\tilde{z} \otimes 1_{G}$. Since the set of all $\tilde{z}$ is weakly dense in $B\left(\mathscr{K} \otimes L^{2}(G)\right)$, (8.11) holds on $M_{a} \otimes 1_{G}$ and hence $\beta$ satisfies (2.6), which shows that $\beta$ is a dual action of $G$ on $M_{\alpha}$.

Finally we shall show that $M$ is isomorphic to $N \otimes_{\beta}^{d} G$, where $N \equiv$ $M_{\alpha}$. Since

$$
w^{*} \beta(x) w=x \otimes 1_{G}=\alpha(x)
$$

for $x$ in $N$ and

$$
\begin{gathered}
\left(w^{*}\left(1_{N} \otimes f\right) w \xi\right)(s, t)=\Delta(t)^{1 / 2}\left(\left(1_{N} \otimes f\right) w \xi\right)\left(s, t^{-1} s\right) \\
=f\left(t^{-1} s\right) \Delta(t)^{1 / 2}(w \xi)\left(s, t^{-1} s\right)=f\left(t^{-1} s\right) \xi(s, t) \\
=\alpha_{t}\left(1_{\mathscr{N}} \otimes f\right) \xi(s, t)=\left(\alpha\left(1_{\mathscr{H}} \otimes f\right) \xi\right)(s, t)
\end{gathered}
$$

for $f$ in $L^{\infty}(G)$, we have $N \otimes_{\beta}^{d} G=\operatorname{Ad} w \circ \alpha(M)$ and hence $M$ is isomorphic to $N \otimes{ }_{\beta}^{d} G$.

Q.E.D.

Theorem 8.3 gives a sufficient condition under which Haga's factorization holds for a crossed product, [7], in the following. 
Theorem 8.4. Let $\alpha$ be an action of $G$ on $M$. If $1_{\mathscr{K}} \otimes L^{\infty}(G)$ is $a$ von Neumann subalgebra of $M$ satisfying $\alpha_{t}\left(1_{\mathscr{x}} \otimes f\right)=1_{\mathscr{K}} \otimes f_{t^{-1}}$ for all $t \in G$, then $M \otimes_{\alpha} G$ is isomorphic to $M^{\alpha} \otimes B\left(L^{2}(G)\right)$.

Proof. By virtue of Theorem 8.3 we have a von Neumann algebra $N$ and a dual action $\beta$ of $G$ on $N$ such that $M$ is isomorphic to $N \otimes_{\beta}^{d} G$. We set $\rho \equiv \operatorname{Ad} w \circ \alpha$. It follows from (8.12) and (8.13) that $\rho(x)=\beta(x)$ for $x \in N \equiv M_{\alpha}$ and $\rho\left(1_{\mathscr{X}} \otimes f\right)=1_{N} \otimes f$ for $f \in L^{\infty}(G)$. Since

$$
\begin{aligned}
& (\rho \otimes \iota)(\alpha(x))=\rho(x) \otimes 1_{G}=\beta(x) \otimes 1_{G} \\
& =\hat{\beta}(\beta(x))=\hat{\beta}(\rho(x))
\end{aligned}
$$

for $x \in N \equiv M_{\alpha}$ and

$$
\begin{aligned}
& (\rho \otimes \ell) \alpha\left(1_{\mathscr{H}} \otimes f\right)=\left(\rho \otimes_{\ell}\right)\left(1_{\mathscr{H}} \otimes \varepsilon f\right)=1_{N} \otimes \varepsilon f \\
& =\hat{\beta}\left(1_{N} \otimes f\right)=\hat{\beta}\left(\rho\left(1_{\mathscr{K}} \otimes f\right)\right),
\end{aligned}
$$

we know that $(\rho \otimes \imath) \circ \alpha=\hat{\beta} \circ \rho$ and hence $\alpha$ is an action dual to $\beta$ through the isomorphism $\rho$. Therefore, by Corollary 7.4, we have a desired result.

Q.E.D.

\section{Appendix}

In this section we shall give a few comments on our results considered when we use the left regular representation of $G$ on $L^{2}(G)$. Let $J$ be a unitary involution on $L^{2}(G)$ defined by $(J \xi)(t) \equiv \Delta(t)^{1 / 2} \xi\left(t^{-1}\right)$.

We set

$$
\begin{array}{ll}
\alpha^{\prime} \equiv\left(\operatorname{Ad} 1_{M} \otimes J\right) \circ \alpha, & \delta^{\prime} \equiv(\operatorname{Ad} J \otimes J) \circ \delta \circ(\operatorname{Ad} J)^{-1} \\
\beta^{\prime} \equiv\left(\operatorname{Ad~} 1_{N} \otimes J\right) \circ \beta, & \gamma^{\prime} \equiv(\operatorname{Ad} J \otimes J) \circ \gamma \circ(\operatorname{Ad} J)^{-1} \\
M \otimes_{\alpha^{\prime}} G \equiv\left\{\alpha^{\prime}(M), 1_{M} \otimes R(G)^{\prime}\right\}^{\prime \prime}, & N \otimes_{\beta^{\prime}}^{d} G \equiv\left\{\beta^{\prime}(N), 1_{N} \otimes L^{\infty}(G)\right\}^{\prime \prime} .
\end{array}
$$

Then we have

$$
\begin{aligned}
& \left(\alpha^{\prime} \otimes \imath\right) \circ \alpha^{\prime}=\left(\iota \otimes \delta^{\prime}\right) \circ \alpha^{\prime}, \quad\left(\alpha^{\prime}(x) \xi\right)(t)=\alpha_{t}^{-1}(x) \xi(t) \\
& \left(\beta^{\prime} \otimes \iota\right) \circ \beta^{\prime}=\left(\iota \otimes \gamma^{\prime}\right) \circ \beta^{\prime}
\end{aligned}
$$




$$
\begin{array}{ll}
\left(\delta^{\prime} f\right)(s, t)=f(t s), & \gamma^{\prime} \lambda^{\prime}(t)=\lambda^{\prime}(t) \otimes \lambda^{\prime}(t) \\
M \otimes_{\alpha^{\prime}} G \sim M \otimes_{\alpha} G, & N \otimes_{\beta^{\prime}}^{d} G \sim N \otimes_{\beta}^{d} G .
\end{array}
$$

If we define $\hat{\alpha}^{\prime}$ and $\hat{\beta}^{\prime}$ by

$$
\hat{\alpha}^{\prime}(y) \equiv \operatorname{Ad} 1_{M} \otimes W^{\prime}\left(y \otimes 1_{G}\right), \quad \hat{\beta}^{\prime}(z) \equiv \operatorname{Ad} 1_{N} \otimes V^{*}\left(z \otimes 1_{G}\right)
$$

for $y \in M \otimes_{\alpha^{\prime}} G$ and $z \in N \otimes_{\beta^{\prime}}^{d} G$, then

$$
\begin{array}{ll}
\hat{\alpha}^{\prime}\left(\alpha^{\prime}(x)\right)=\alpha^{\prime}(x) \otimes 1_{G}, & \hat{\alpha}^{\prime}\left(1_{M} \otimes \lambda^{\prime}(r)\right)=1_{M} \otimes \lambda^{\prime}(r) \otimes \lambda^{\prime}(r) \\
\hat{\beta}^{\prime}\left(\beta^{\prime}(x)\right)=\beta^{\prime}(x) \otimes 1_{G}, & \hat{\beta}^{\prime}\left(1_{N} \otimes f\right)=1_{N} \otimes \varepsilon^{\prime} f,
\end{array}
$$

where $\left(\varepsilon^{\prime} f\right)(s, t) \equiv f\left(s t^{-1}\right)$. Besides, $\hat{\alpha}^{\prime}$ and $\hat{\beta}^{\prime}$ satisfying (9.2) and (9.1), respectively.

\section{References}

[1] Araki, H., Structure of some von Neumann algebras with isolated discrete modular spectrum, Publ. RIMS, Kyoto Univ., 9 (1973), 1-14.

[2] Arveson, W. B., On groups of automorphisms of operator algebras, J. Functional Analysis, 15 (1974), 217-243.

[3] Connes, A., Une classification des facteurs de type III, Ann. Sci. École Norm. Sup., 6 (1973), 133-252.

[4] Doplicher, S., Haag, R. and Roberts, J. E., Fields, observables and gauge transformations I, Commun. math. Phys., 13 (1969), 1-23.

[5] Eymard, P., L'algèbre de Fourier d'un groupe localement compact, Bull. Soc. Math. France, 92 (1964), 181-236.

[6] Haagerup, U., Operator valued weights and crossed product, Preprint of Univ. Odense, Denmark, 1974.

[7] Haga, Y., Crossed products of von Neumann algebras by compact groups, Tôhoku Math. J., 28 (1976), 511-522.

[8] Ikunishi, A. and Nakagami, Y., On invariants $G(\sigma)$ and $\Gamma(\sigma)$ for an automorphism group of a von Neumann algebra, Publ. RIMS, Kyoto Univ., 12 (1976), 1-30.

[9] Landstad, M. B., Duality theory for covariant systems, Preprint of Univ. Trondheim, Norway, 1974.

[10] Mackey, G. W., A theorem of Stone and von Neumann, Duke Math. J., 16 (1949), 313-326.

[11] Moore, R. T., Measurable, continuous and smooth vectors for semi-groups and group representations, Memoirs of Amer. Math. Soc., 78 (1968), 1-80.

[12] Murakami, K., On the $W^{*}$-tensor product of $L^{\infty}(G)$ and $M$, Preprint of Tokyo Inst. Tech., Japan, 1975.

[13] Nakagami, Y., Duality for crossed products of von Neumann algebras by local- 
ly compact groups, Bull. Amer. Math. Soc., 81 (1975), 1106-1108.

[14] Roberts, J. E., Cross products of von Neumann algebras by group duals, Preprint of C.N. R.S., Marseille, France, 1975.

[15] Saito, K., On a duality for locally compact groups, Tôhoku Math. J., 20 (1968), 355-367.

[16] Takemoto, H., On the weakly continuous constant field of Hilbert space and its application to the reduction theory of $W^{*}$-algebra, Tôhoku Math. J., to appear.

[17] Takesaki, M., Duality and von Neumann algebras, Lecture on Operator Algebras (Edited by K. H. Hofmann), Lecture notes in Math., 247 (1972), 666-779, Springer-Verlarg, Berlin.

[18] Takesaki, M., Duality for crossed products and the structure of von Neumann algebras of type III, Acta Math., 131 (1973), 249-310.

[19] Tomiyama, J., Tensor products and projections of norm one in von Neumann algebras, Lecture note at Univ. Copenhagen, Denmark, 1970.

[20] Strătilă, S., Voiculescu, D. and Zsidó, L., Sur les produits croises, Preprint of INCREST, Bucharest, 1975.

[21] Landstad, M. B., Duality for dual covariance algebra, Preprint of Univ. Trondheim, Norway, 1976.

[22] Strătilă, S., Voiculescu, D. and Zsidó, L., On crossed products, Preprint of INCREST, Bucharest, 1976.

Acknowledgement. After completing this paper the author has received preprints $[20,21,22]$ containing essentially the same results. $\mathrm{He}$ appreciates these preprints and their private communications. Especially, Strǎtilă, Voiculescu and Zsidó have pointed out an error in Lemma 4.5 .

Correction. In [13] the unitary $V$ in Theorem 2 should be read as $U$ defined below, and Theorem 3 should be replaced by Corollary without assuming the unimodularness. $\alpha$ in Theorem 4 shoud be read $\hat{\beta}$. 
